Ref. SISSA 152/1999/FM

nlin.SI/0001052

\title{
Super KP equations and Darboux transformations: another perspective on the Jacobian Super KP hierarchy
}

\author{
Gregorio Falqui, Cesare Reina and Alessandro Zampa \\ SISSA, Via Beirut 2/4, I-34014 Trieste, Italy \\ E-mail: falqui@sissa.it, reina@sissa.it, zampa@fm.sissa.it
}

\begin{abstract}
We generalize to the supersymmetric case the representation of the KP hierarchy as a set of conservation laws for the generating series of the conserved densities. We show that the hierarchy so obtained is isomorphic to the JSKP of Mulase and Rabin. We identify its "bosonic content" with the so-called Darboux-KP hierarchy, which geometrically encompasses the theory of Darboux-Bäcklund transformations, and is an extension both of the KP theory and of the modified KP theory. Finally, we show how the hierarchy can be linearized and how the supersymmetric counterpart of a wide class of rational solution can be quite explicitly worked out.
\end{abstract}

\section{Introduction}

Supersymmetric extensions of integrable hierarchies of PDE's are by now a wellstudied subject (see, e.g., [17, 25, 30, 32, 26, 20, 19, 21]). In particular, for the KP equations, two different extensions have emerged: the Manin-Radul SKP [25] (MRSKP), and the Jacobian SKP of Mulase and Rabin [30, 31] (JSKP). The most remarkable and best know differences between the two hierarchies are the fact that the flows of MRSKP are a representation of a super Heisenberg algebra, while those of JSKP are supercommuting ones, and the behavior of their algebro-geometric solution. Indeed, the JSKP describes linear flows on the (super) Jacobian manifold of a

super algebraic curve $\widehat{C}$, while the MRSKP flows involve, in general, a motion on the space of moduli of $\widehat{C}$. Actually, the latter is perhaps the strongest motivation that 
led Mulase and Rabin to modify the (previously discovered) Manin-Radul supersymmetric extension of the KP hierarchy.

From the point of view of the present paper, it is more relevant another difference between the two approaches: the Manin-Radul theory concerns the supersymmetric extension of the Lax representation:

$$
\frac{\partial L}{\partial t_{k}}=\left[\left(L^{k}\right)_{+}, L\right]
$$

of the KP hierarchy on the space of pseudo-differential operators in one dimension. In the approach of Mulase and Rabin, one starts instead from the Sato representation of the KP equations on the Volterra group of Dressing operators,

$$
\frac{\partial S}{\partial t_{k}}=-\left(S \partial_{x}^{k} S^{-1}\right)_{-} S
$$

where $S$ and $L$ are related by the well-know dressing formula

$$
L=S \partial_{x} S^{-1}
$$

This paper is based on the representation of the KP theory as a set of conservation laws [39], which has been recently studied [10] as an outgrowth of the application of the Gel'fand-Zakharevich theorem [13] to infinite dimensional integrable systems. Namely, the KP equations are written as the conservation laws

$$
\frac{\partial h}{\partial t_{k}}=\partial_{x} H^{(k)}
$$

where

$$
h=z+\sum_{l=1}^{\infty} \frac{h_{l}}{z^{l}}
$$

is the generating series of the conserved densities of the KP theory, and $H^{(k)}$ are their current densities. These currents are written as suitable linear combinations of the Faà di Bruno monomials

$$
h^{(k)}=\left(\partial_{x}+h\right)^{k} \cdot 1
$$

associated with $h$.

The supersymmetric extension of the equations (1.4) will be written as

$$
\frac{\partial \hat{h}}{\partial t_{\alpha}}=(-1)^{\alpha} \delta \widehat{H}^{(\alpha)}
$$

and called Hamiltonian Super KP hierarchy (HSKP). Here $\delta=\partial_{\varphi}+\varphi \partial_{x}$ is the square root on the super-circle $S^{1 \mid 1}$ of the $x$-derivative $\partial_{x}$, well known from string and superconformal field theories (see, e.g., [12]), the odd superfield $\hat{h}$ replaces the 
generator $h$ of the local hamiltonian densities, and $\widehat{H}^{(\alpha)}$ are suitable supersymmetric extensions of the currents $H^{(j)}$. In the first part of the paper we show that, as it happens in the ordinary case, equations (1.7) admit a representation-extension to a family of dynamical systems with $\mathbb{N}^{2} \times \mathbb{N}^{2}$ variables (of which half are even and half are odd), to be called the Super Central System (SCS). It is a counterpart of the Sato system on the infinite-dimensional Grassmann manifold [33, 7, 35, 28, 37], and the Super KP equations (1.7) can be considered as a kind of "reduction" of SCS. Then we show how the hierarchy $(1.7)$ can be identified, by means of a non trivial coordinate change, with the Jacobian Super KP hierarchy.

This is however only the first topic we want to discuss in this paper. The second one concerns the relation of the HSKP hierarchy with the theory of Darboux transformations. The fact that supersymmetry has an intriguing relation with the theory of Darboux transformations is a well established one. For instance, the classical problem of factorizing the Schrödinger operator $-\partial_{x}^{2}+u(x)$ into first order factors gives rise to a super algebra (see [27], §2, and references quoted therein). Another signal of this fact comes from Mulase's paper [29], where it was shown how the modified KP equation can be obtained from the (Manin-Radul) SKP by means of a process of elimination of odd variables.

We shall show that HSKP provides a natural framework to discuss such issues. We shall use the geometrical setting of [22], where the classical subject of DarbouxBäcklund transformations and Miura-like maps is approached in a rather unconventional way that can be summarized as follows. Instead of searching directly for a symmetry of an evolutionary equation $X$ defined on a manifold $M$, one tries to find a covering, that is another evolutionary equation, defined on a bigger manifold $N$, related to $X$ by two maps $\pi, \sigma: N \rightarrow M$, such that

$$
X=\pi_{*} Y=\sigma_{*} Y
$$

In 222 a covering for the KP hierarchy, called Darboux-KP (DKP) hierarchy, was constructed as a hierarchy defined on the phase space of pairs of Laurent series $(h, a)$ with suitable asymptotics and the (generalized) Miura transformation was defined to be $h \mapsto \sigma(a, h)=h+a_{x} / a$. Another application of this formalism has been used in [10] to linearize the equations (the so called Central System (CS)) induced by the flows (1.4) on the currents $H^{(j)}$. This method has been exploited (see [11]) to construct a wide class of solutions of KP admitting a polynomial $\tau$-function.

In this paper we will consider the supersymmetric counterpart of the geometric theory of the Darboux transformations. Firstly, we will show that the DKP system is actually the bosonic content of the even flows of HSKP (and hence of JSKP). Then we will construct a Darboux covering for HSKP and related "rational" reductions. Finally, we will use the technique of Darboux covering to linearize the Super analogue SCS of CS, exploiting this result in a quite explicit description of a wide class of non trivial solutions of HSKP of rational type.

The detailed plan of the paper is the following: in Section 2, after having briefly recalled the basis of the (bi)hamiltonian set up for the KP theory, we will introduce the phase space for HSKP and define the hierarchy. In Section 2.2 we will discuss the 
fundamental properties of HSKP, and we will introduce the Super Central System SCS as the dynamical system obeyed by the currents of the theory when the Faà di Bruno generator $\hat{h}$ evolves along HSKP. In Section 2.3 we will show how solutions of HSKP can be obtained from solutions of SCS, and in Section 2.4 we will briefly discuss how HSKP can be seen as a particular form of the Jacobian SKP hierarchy of Mulase and Rabin, by comparing the wave functions associated with the two theories. In Section 2.5, we will show how a super extension of the KdV equation can be obtained as a suitable reduction of HSKP; this will give us a concrete clue to the rest of the paper. Indeed, from Section 3 onwards we will turn our attention to the method of Darboux coverings. We will first recall the setting of the ordinary bosonic case, and then identify the bosonic part of HSKP with the DKP hierarchy of [22]. We will also point out the specific form of the generalized Miura transformation. Furthermore, we will construct a Darboux covering of HSKP, and briefly discuss some reductions of the latter. Finally, in Section 1 we will show how the equations can be explicitly linearized, and discuss a specific class of solutions depending rationally on a finite number of times.

\section{The GZ approach to KP and its supersymmetric extension}

The technique that plays a prominent role in the bi-Hamiltonian approach to KP is the Gelfan'd Zakharevich method of Poisson pencils to construct integrable Hamiltonian systems [13]. In such a scheme one considers a manifold $M$ endowed with a pencil $P_{\lambda}=P_{1}-\lambda P_{0}$ of Poisson structures, and studies the Casimir functions of the pencil. Such a Casimir function $H_{\lambda}$ is a (non-constant) function on $M$, which depends also on the parameter $\lambda$, such that $P_{\lambda} d H_{\lambda}=0$ for every value of $\lambda$. When $M$ is an $2 n+1$-dimensional manifold endowed with a Poisson pencil of maximal rank, $P_{\lambda}$ has a unique Casimir function $H_{\lambda}$, which is a polynomial in $\lambda$ of degree $n$,

$$
H_{\lambda}=H_{0} \lambda^{n}+H_{1} \lambda^{n-1}+\cdots+H_{n} .
$$

Its leading coefficient $H_{0}$ is the Casimir of $P_{0}$, while the "constant term" $H_{n}$ is the Casimir of $P_{1}$. The coefficients $H_{j}$ satisfy the recurrence relations

$$
P_{1} d H_{j+1}=P_{0} d H_{j}
$$

and therefore are in involution with respect to all the brackets of the pencil.

In the realm of infinite dimensional systems, the $\mathrm{KdV}$ theory is perhaps the best known prototype of a GZ hierarchy [6, 14]. Here, the manifold $M$ is the space of $C^{\infty}$ functions on the circle $S^{1}$, and the Poisson pencil, given as a one-parameter family of skew-symmetric maps from the cotangent to the tangent bundle, reads

$$
\dot{u}=\left(P_{\lambda}\right)_{u} v=-\frac{1}{2} v_{x x x}+2(u+\lambda) v_{x}+u_{x} v
$$


where $x$ is a coordinate on $S^{1}, u$ represents a point of $M$, and $\dot{u}$ and $v$ are respectively a vector and a a covector at $u$. It turns out [10 that if $h$ and $v$ are series in $z=\sqrt{\lambda}$ of the form

$$
h(z)=z+\sum_{j>0} h_{j} z^{-j}, \quad v=1+\sum_{l>0} v_{l} / z^{2 l}
$$

that provide the unique solutions of the Riccati system

$$
\left\{\begin{array}{l}
h_{x}+h^{2}=u+z^{2} \\
-1 / 2 v_{x}+h v=z
\end{array},\right.
$$

then $v(z)$ is the series representing the differential of the Casimir function of the Poisson pencil of $\mathrm{KdV}$, which, in turn, is given by the integral

$$
H_{\lambda}=2 z \int_{S^{1}} h d x
$$

The GZ hierarchy associated with $H_{\lambda}$ on $M$ admits several representations. The one we are interested in can be expressed by saying that the local Hamiltonian density $h(z)$ must obey local conservation laws of the form

$$
\frac{\partial}{\partial t_{j}} h=\partial_{x} H^{(j)}
$$

where the "current densities" $H^{(j)}$ are given by

$$
H^{(2 j)}=\lambda^{j} \text { and } \quad H^{(2 j+1)}=-\frac{1}{2}\left(\lambda^{j} v\right)_{+, x}+h\left(\lambda^{j} v\right)_{+}
$$

the subscript + meaning to take the positive part of the expansion in powers of $z$. Equation (2.3) can also be written as

$$
H^{(2 j+1)}=z^{2 j}\left(-\frac{1}{2} v_{x}+h v\right)+\frac{1}{2}\left(z^{2 j} v\right)_{-, x}-h\left(z^{2 j} v\right)_{-}
$$

or

$$
H^{(2 j+1)}=\sum_{l=1}^{j}\left[-\frac{1}{2} v_{j-l, x}\left(z^{2 l} \cdot 1\right)+v_{j-l}\left(z^{2 l} \cdot h\right)\right] .
$$

The first of these two expressions shows that $H^{(j)}=z^{j}+O\left(z^{-1}\right)$, since by the second Riccati equation above we have $z^{2 j}\left(-\frac{1}{2} v_{x}+h v\right)=z^{2 j+1}$. The interpretation of the second entails that the currents $H^{(j)}$ are the unique combinations

$$
H^{(j)}=\sum_{k=0}^{j} c_{k}^{j} h^{(k)}
$$

of the Faà di Bruno iterates $h^{(j)}$ of $h^{(0)}=1$ at $h$, defined by

$$
h^{(j+1)}=\left(\partial_{x}+h\right) h^{(j)},
$$


that admit the asymptotic expansion $H^{(j)}=z^{j}+O\left(z^{-1}\right)$.

The relevance of this result is that the currents $H^{(j)}$ can be constructed without requiring that $h$ is a solution of the Riccati equation. Therefore one can define the KP hierarchy as follows.

Definition 2.1 Let $h$ be a monic (formal) Laurent series in $z^{-1}$

$$
h:=z+\sum_{j>0} h_{j} z^{-j}
$$

whose coefficients $h_{j}$ belong to $C^{\infty}\left(S^{1}\right)$, and consider its Fà̀ di Bruno iterates $h^{(j)}$. Denote by $W$ the span over $C^{\infty}\left(S^{1}\right)$ of the order Fa⿳亠 di Bruno iterates (or monomials)

$$
W:=\operatorname{span}_{C^{\infty}\left(S^{1}\right)}\left\{h^{(k)}, k \geq 0\right\}
$$

and introduce the "current densities" $H^{(k)}$ by requiring them to be the unique elements of $W$ of the form

$$
H^{(k)}=z^{k}+\sum_{j>0} H_{j}^{k} z^{-j} .
$$

The KP hierarchy is defined to be the set of conservation laws

$$
\frac{\partial}{\partial t_{k}} h=\partial_{x} H^{(k)}
$$

We observe that $H^{(1)}=h$, so we can identify the first time $t_{1}$ with $x$. Moreover, it can be proven that this definition is completely equivalent to the one given in the framework of pseudo-differential operators (see, e. g., [8] and references quoted therein).

We end this review of the bihamiltonian set-up of the KP hierarchy with the notion of the Central System (CS). The operators $\partial_{t_{k}}+H^{(k)}$ satisfy the invariance condition

$$
\left(\partial_{t_{k}}+H^{(k)}\right) W \subset W
$$

and the commutativity property $\left[\partial_{t_{k}}+H^{(k)}, \partial_{t_{j}}+H^{(j)}\right]=0$. This entails that along the KP flows (2.5) the currents $H^{(k)}$ satisfy the following evolutionary equation:

$$
\frac{\partial}{\partial t_{j}} H^{(k)}+H^{(j)} H^{(k)}=H^{(j+k)}+\sum_{l=1}^{k} H_{l}^{j} H^{(k-l)}+\sum_{l=1}^{j} H_{l}^{k} H^{(j-l)} .
$$

Definition 2.2 Let $\mathcal{H}$ be the space of sequences $\left\{H^{(0)}, H^{(1)}, H^{(2)}, H^{(3)}, \ldots\right\}$ where $H^{(0)}=1$ and the $H^{(j)}$ 's are of the form:

$$
H^{(j)}=z^{j}+\sum_{l \geq 1} \frac{H_{l}^{j}}{z^{l}}, j \geq 1
$$

The Central System is the hierarchy of dynamical system on $\mathcal{H}$ defined by equation (2.0). It turns out that the vector fields of CS commute among themselves [4. 
In the next Section we define the extension to the $(N=1)$ supersymmetric case of the constructions herewith outlined. This will lead us to the definition of a supersymmetric extension of the KP hierarchy. We will refer to such a SKP theory as Hamiltonian Super KP, to keep track of its (albeit remote) hamiltonian origins. Later (see Section 2.4) we will show how to identify our HSKP with the Jacobian super KP hierarchy of Mulase and Rabin [30, 32, 2, 36]) .

\subsection{The definition of the Hamiltonian super KP hierarchy}

Let us start by fixing some notations (see, e.g., [24] for more details on supergeometry), to be used throughout the paper. We denote by $\Lambda$ a generic Grassmann algebra over $\mathbb{C}$. This is required by functorial properties of supersymmetry [34], but in this work it will play a spectator role, and can be thought of as a fixed algebra. We supplement the bosonic spectral parameter $z$ with its fermionic "super-partner" $\theta$, and replace the circle by its super analog $S^{1 \mid 1}$ endowed with coordinates $x, \varphi$. To simplify notations we call $B_{[x \varphi]}$ the ring $C^{\infty}\left(S^{1 \mid 1}, \Lambda\right)$ of smooth functions on $S^{1 \mid 1}$ with values in $\Lambda$ (or a suitable "version" of it, like the space of $\Lambda$-valued functions on $\mathbb{R}^{1 \mid 1}$ vanishing at infinity, or even the space $\mathbb{C}[[x, \varphi]] \otimes \Lambda$ of formal series in $x, \varphi$.) Finally, we denote by $\bar{f}$ the parity of a homogeneous element $f$, e.g. $\bar{z}=\bar{x}=0, \bar{\theta}=\bar{\varphi}=1$.

The Hamiltonian super KP hierarchy is defined in terms of the super Faà di Bruno generator $\hat{h}$ and the odd derivation operator $\delta:=\partial_{\varphi}+\varphi \partial_{x}$ taking the place of $\partial_{x}$. The generator $\hat{h}$ is an odd formal Laurent series in $z^{-1}$ and $\theta$ with coefficients in $B_{[x \varphi]}$ of the form

$$
\hat{h}(z, \theta ; x, \varphi):=\nu(z ; x)+\theta a(z ; x)+\varphi h(z ; x)+(\theta \varphi) \psi(z ; x),
$$

We specify exactly the content of the components $\nu, a, h$ and $\psi$, by analogy with the $\mathrm{KP}$ formalism, requiring that:

1. All equations are homogeneous with respect to the grading specified by

$$
[\theta]=\frac{1}{2},[z]=1,[\varphi]=-\frac{1}{2},[x]=-1,[\hat{h}]=[\delta]=\frac{1}{2},\left[t_{j}\right]=\frac{j}{2},
$$

and no field of negative weight enters the theory.

2. It is possible to identify the second time $t_{2}$ with $x$.

3. There exist suitable "super current densities" $\widehat{H}^{(k)}$, with asymptotic behavior

$$
\widehat{H}^{(2 j+p)} \sim z^{j} \theta^{p}+O(1 / z), j \in \mathbb{N}, p \in\{0,1\}
$$

It turns out that these requirements can be satisfied if the following simple and convenient choices are made:

\footnotetext{
1 The relation of $t_{1}$ with $\varphi$ will be however much subtler. We will discuss it in Section 2.3.
} 
i) $a$ is holomorphic in $z^{-1}$ with constant and invertible zeroth order coefficient (which we assume to be equal to 1$): a(z ; x):=1+\sum_{j>0} a_{j}(x) z^{-j}$.

ii) $h$ has the usual form $h(z ; x):=z+\sum_{j>0} h_{j}(x) z^{-j}$.

iii) $\nu$ and $\psi$ are of the form

$$
\nu(z ; x):=\sum_{j>0} \nu_{j}(x) z^{-j}, \quad \psi(z ; x):=\sum_{j>0} \psi_{j}(x) z^{-j} .
$$

With the super Faà di Bruno generator $\hat{h}$ we associate (for $k \in \mathbb{N}$ ) its iterates

$$
\left\{\begin{array}{l}
\hat{h}^{(k+1)}:=(\delta+\hat{h}) \cdot \hat{h}^{(k)} \\
\hat{h}^{(0)}:=1
\end{array} .\right.
$$

The following lemma shows that the even Faà di Bruno iterates, apart from their nilpotent components, are essentially the usual Faà di Bruno monomials.

Lemma 2.1 Let $\hat{f}:=\delta(\hat{h})=h-\theta \psi+\varphi \nu_{x}-(\theta \varphi) a_{x}$. Then, for any $k \in \mathbb{N}$

$$
\left\{\begin{array}{l}
\hat{h}^{(2 k+2)}=\left(\partial_{x}+\hat{f}\right) \cdot \hat{h}^{(2 k)}=\left(\partial_{x}+\hat{f}\right)^{k+1} \cdot 1 \\
\hat{h}^{(2 k+3)}=\left(\partial_{x}+\hat{f}\right) \cdot \hat{h}^{(2 k+1)}=\left(\partial_{x}+\hat{f}\right)^{k+1} \cdot \hat{h}
\end{array} .\right.
$$

Proof. We have

$$
(\delta+\hat{h})^{2}=\delta^{2}+\delta(\hat{h})-\hat{h} \delta+\hat{h} \delta+\hat{h}^{2}=\delta^{2}+\delta(\hat{h})=\partial_{x}+\hat{f},
$$

so we get

$$
\hat{h}^{(2 k+2)}=(\delta+\hat{h})^{2} \cdot \hat{h}^{(2 k)}=\left(\partial_{x}+\hat{f}\right) \cdot \hat{h}^{(2 k)}=(\delta+\hat{h})^{2 k+2} \cdot 1=\left(\partial_{x}+\hat{f}\right)^{k+1} \cdot 1
$$

and

$$
\hat{h}^{(2 k+3)}=(\delta+\hat{h})^{2} \cdot \hat{h}^{(2 k+1)}=\left(\partial_{x}+\hat{f}\right) \cdot \hat{h}^{(2 k+1)}=(\delta+\hat{h})^{2 k+2} \cdot \hat{h}=\left(\partial_{x}+\hat{f}\right)^{k+1} \cdot \hat{h} .
$$

For later use we express the Faà di Bruno iterates as

$$
\left\{\begin{array}{l}
\hat{h}^{(2 k)}=h^{(k)}-\theta \psi^{(k)}+\varphi \omega^{(k)}-(\theta \varphi) b^{(k)} \\
\hat{h}^{(2 k-1)}=\nu^{(k)}+\theta a^{(k)}+\varphi d^{(k)}+(\theta \varphi) \chi^{(k)}
\end{array},\right.
$$

where the components are Laurent series of the form

$$
\begin{array}{ll}
\nu^{(k)}=\sum_{j>0} \nu_{j}^{(k)} z^{k-j-1}, & h^{(k)}=z^{k}+\sum_{j>0} h_{j}^{(k)} z^{k-j-1} \\
a^{(k)}=z^{k-1}+\sum_{j>0} a_{j}^{(k)} z^{k-j-1}, & \psi^{(k)}=\sum_{j>0} \psi_{j}^{(k)} z^{k-j-1} \\
d^{(k)}=z^{k}+\sum_{j>0} d_{j}^{(k)} z^{k-j-1}, & \omega^{(k)}=\sum_{j>0} \omega_{j}^{(k)} z^{k-j-1} \\
\chi^{(k)}=\sum_{j>0} \chi_{j}^{(k)} z^{k-j-1}, & b^{(k)}=\sum_{j>0} b_{j}^{(k)} z^{k-j-1}
\end{array}
$$

and can be computed by recurrence according to the rules displayed in Table 1 


\begin{tabular}{|c|c|}
\hline Table 1 & \\
\hline$\left\{\begin{array}{l}\nu^{(k+1)}=\left(\partial_{x}+h\right) \nu^{(k)} \\
\nu^{(1)}=\nu\end{array}\right.$ & $\left\{\begin{array}{l}h^{(k+1)}=\left(\partial_{x}+h\right) h^{(k)} \\
h^{(0)}=1\end{array}\right.$ \\
\hline$\left\{\begin{array}{l}a^{(k+1)}=\left(\partial_{x}+h\right) a^{(k)}-\psi \nu^{(k)} \\
a^{(1)}=a\end{array}\right.$ & $\left\{\begin{array}{l}\psi^{(k+1)}=\left(\partial_{x}+h\right) \psi^{(k)}+\psi h^{(k)} \\
\psi^{(0)}=0\end{array}\right.$ \\
\hline$\left\{\begin{array}{l}d^{(k+1)}=\left(\partial_{x}+h\right) d^{(k)}+\nu_{x} \nu^{(k)} \\
d^{(1)}=h\end{array}\right.$ & $\left\{\begin{array}{l}\omega^{(k+1)}=\left(\partial_{x}+h\right) \omega^{(k)}+\nu_{x} h^{(k)} \\
\omega^{(0)}=0\end{array}\right.$ \\
\hline$\left\{\begin{aligned} \chi^{(k+1)}= & \left(\partial_{x}+h\right) \chi^{(k)}+\psi d^{(k)} \\
& +\nu_{x} a^{(k)}-a_{x} \nu^{(k)} \\
\chi^{(1)}=\psi & \end{aligned}\right.$ & $\left\{\begin{aligned} b^{(k+1)}= & \left(\partial_{x}+h\right) b^{(k)}-\psi \omega^{(k)} \\
& -\nu_{x} \psi^{(k)}+a_{x} h^{(k)} \\
b^{(0)}=0 & \end{aligned}\right.$ \\
\hline
\end{tabular}

By analogy with the KP hierarchy, we introduce the space

$$
W_{B_{[x \varphi]}}:=\operatorname{span}_{B_{[x \varphi]}}\left\{\hat{h}^{(k)}, k \in \mathbb{N}\right\}
$$

and prove the existence of the super currents with the desired asymptotic behavior.

Proposition 2.2 Let $\hat{h}$ and $W_{B_{[x \varphi]}}$ be defined as in equations (2.X) and (2.19). There exists a basis $\left\{\widehat{H}^{(k)}, k \in \mathbb{N}\right\}$ of $W_{B_{[x \varphi]}}$ with

$$
\left\{\begin{array}{l}
\widehat{H}^{(2 k)}=z^{k}+\sum_{j>0}\left(\widehat{H}_{0, j}^{2 k}(x, \varphi) z^{-j}+\widehat{H}_{1, j}^{2 k}(x, \varphi) \theta z^{-j}\right) \\
\widehat{H}^{(2 k+1)}=\theta z^{k}+\sum_{j>0}\left(\widehat{H}_{0, j}^{2 k+1}(x, \varphi) z^{-j}+\widehat{H}_{1, j}^{2 k+1}(x, \varphi) \theta z^{-j}\right)
\end{array} .\right.
$$

Proof. By definition of $W_{B_{[x \varphi]}}$ we see that

$$
\left\{\begin{array}{l}
\widehat{H}^{(0)}=1 \\
\widehat{H}^{(1)}=\hat{h}^{(1)}-\varphi \hat{h}^{(2)} \\
\widehat{H}^{(2)}=\hat{h}^{(2)}
\end{array}\right.
$$

The others can be computed recursively: suppose we have defined $\widehat{H}^{(j)}$ for $0 \leq j<k$; if $k=2 n$ is even then

$$
\widehat{H}^{(k)}=\hat{h}^{(k)}-\sum_{j=1}^{n-1}\left(h_{j}^{(n)}+\varphi \omega_{j}^{(n)}\right) \widehat{H}^{(k-2 j-2)}-\sum_{j=1}^{n-1}\left(\psi_{j}^{(n)}+\varphi b_{j}^{(n)}\right) \widehat{H}^{(k-2 j-1)},
$$


while if $k=2 n-1$ is odd then

$$
\begin{aligned}
\widehat{H}^{(k)}= & \hat{h}^{(k)}-\varphi \hat{h}^{(k+1)}-\sum_{j=1}^{n-1}\left(\nu_{j}^{(n)}+\varphi\left(d_{j}^{(n)}-h_{j}^{(n)}\right)\right) \widehat{H}^{(k-2 j-1)} \\
& -\sum_{j=1}^{n-1}\left(a_{j}^{(n)}+\varphi\left(\chi_{j}^{(n)}-\psi_{j}^{(n)}\right)\right) \widehat{H}^{(k-2 j)}
\end{aligned}
$$

We have thus prepared all the "ingredients" needed for the following

Definition 2.3 (HSKP) Let $\hat{h}$ be defined by (2.7), and compute its Fà̀ di Bruno iterates $\hat{h}^{(k)}$ and the basis $\left\{\widehat{H}^{(k)}, k \geq 0\right\}$ of $W_{B_{[x \varphi]}}$ as explained in Proposition 2.2. The Hamiltonian super KP hierarchy is the set of "super conservation laws"

$$
\partial_{t_{k}} \hat{h}=(-1)^{k} \delta \widehat{H}^{(k)}, \quad k>0 .
$$

Notice that, according to the last line of (2.11), one has $\widehat{H}^{(2)}=\hat{h}^{(2)}=\delta \hat{h}$. Hence the $t_{2}$ equation of motion of HSKP is

$$
\partial_{t_{2}} \hat{h}=\delta \widehat{H}^{(2)}=\delta(\delta \hat{h})=\partial_{x} \hat{h}
$$

that is, indeed, $t_{2}$ can be identified with $x$.

\subsection{The super central system}

The first property to be verified is the compatibility of the evolution equations (2.14). While checking this, we shall find that the hierarchy has some very useful properties which allow us to describe it as producing flows on the super universal Grassmannian. This will be accomplished by the introduction of a dynamical system tightly connected with HSKP. Let us first work out some simple consequences of the definition of the hierarchy.

The evolution equations (2.14) are simply the super-commutativity conditions

$$
\left[\delta+\hat{h}, \partial_{t_{k}}+\widehat{H}^{(k)}\right]=0
$$

and imply that

$$
\left(\partial_{t_{k}}+\widehat{H}^{(k)}\right) \cdot W_{B_{[x \varphi]}} \subset W_{B_{[x \varphi]}} .
$$

Indeed,

$$
\begin{aligned}
\left(\partial_{t_{k}}+\widehat{H}^{(k)}\right) & \cdot \hat{h}^{(l)}=\left(\partial_{t_{k}}+\widehat{H}^{(k)}\right) \cdot(\delta+\hat{h})^{l} \cdot 1 \\
& =(-1)^{k l}(\delta+\hat{h})^{l} \cdot\left(\partial_{t_{k}}+\widehat{H}^{(k)}\right) \cdot 1=(-1)^{k l}(\delta+\hat{h})^{l} \cdot \widehat{H}^{(k)}
\end{aligned}
$$

and by definition $\widehat{H}^{(k)} \in W_{B_{[x \varphi]}},(\delta+\hat{h}) \cdot W_{B_{[x \varphi]}} \subset W_{B_{[x \varphi]}}$. In turn, this implies the "abelian zero curvature" equation:

$$
\partial_{t_{j}} \widehat{H}^{(k)}=(-1)^{j k} \partial_{t_{k}} \widehat{H}^{(j)}
$$


as the following simple argument shows.

Denote by $V_{B_{[x \varphi]}}$ the space of Laurent series in $z^{-1}$ and $\theta$ with coefficients in $B_{[x \varphi]}$ and by $V_{B_{[x \varphi]}^{-}}^{-}$its subspace of formal power series without "constant term", i.e. starting from $z^{-1}$ and $\theta z^{-1}$. We have the decomposition

$$
V_{B_{[x \varphi]}}=W_{B_{[x \varphi]}} \oplus V_{B_{[x \varphi]}^{-}} .
$$

Then, by Equation (2.15), $\partial_{t_{j}} \widehat{H}^{(k)}$ is the $V_{B_{[x \varphi]}}^{-}$-component of $-\widehat{H}^{(j)} \widehat{H}^{(k)}$, while $\partial_{t_{k}} \widehat{H}^{(j)}$ is the $V_{B_{[x \varphi]}}^{-}$-component of $-\widehat{H}^{(k)} \widehat{H}^{(j)}=-(-1)^{j k} \widehat{H}^{(j)} \widehat{H}^{(k)}$, thus proving equations (2.16). Thanks to this property and the commutativity of the operators $\delta$ and $\partial_{t_{j}}$, we obtain the compatibility of the evolution equations

$$
\partial_{t_{j}} \partial_{t_{k}} \hat{h}=(-1)^{j+k} \delta \partial_{t_{j}} \widehat{H}^{(k)}=(-1)^{j k+j+k} \delta \partial_{t_{k}} \widehat{H}^{(j)}=(-1)^{j k} \partial_{t_{k}} \partial_{t_{j}} \hat{h} .
$$

This result finally entails the supercommutativity of the operators $\partial_{t_{k}}+\widehat{H}^{(k)}$,

$$
\left[\partial_{t_{j}}+\widehat{H}^{(j)}, \partial_{t_{k}}+\widehat{H}^{(k)}\right]=0
$$

We notice that it is possible to describe the theory in terms of the super-currents $\widehat{H}^{(k)}$ 's only, avoiding the introduction of the super-space variables $x$ and $\varphi$ and the super-derivative $\delta$ which up to now played a special role. It is by doing this that the super universal Grassmannian arises. Let us first of all recall its definition [34, 2, 38]. Denote by $V:=\Lambda\left(\left(z^{-1}\right)\right) \oplus \Lambda\left(\left(z^{-1}\right)\right) \cdot \theta$ the quotient ring of the ring of formal power series in $z^{-1}$ and $\theta$ overf $\Lambda$, and let $V_{+}:=\Lambda[z, \theta], V_{-}:=\Lambda\left[\left[z^{-1}, \theta\right]\right] \cdot z^{-1} . V$ has a natural filtration

$$
\cdots \subset V_{j-1} \subset V_{j} \subset V_{j+1} \subset \cdots,
$$

where $V_{j}=z^{j+1} V_{-}$, which makes it and its $\Lambda$-submodule $V_{+}$complete topological spaces. Then, the super Grassmannian $S G r_{\Lambda}:=S G r_{\Lambda}\left(V, V_{+}\right)$is the set of closed free $\Lambda$-submodules $W$ of $V$ which are compatible with $V_{+}$in the sense that the restriction $\pi_{W}$ of the projection $\pi_{W}: V \rightarrow V_{+}$to $W$ is a Fredholm operator, i.e. its kernel (respectively cokernel) is a $\Lambda$-submodule (respectively a $\Lambda$-quotient module) of a finite rank free $\Lambda$-module. As usual [34, 35], $S G r_{\Lambda}$ is the disjoint union of the denumerable set of its components labelled by the index $i_{W}$ of $\pi_{W}$.

We exploit our formulæ and the concept of super universal Grassmannian by means of the following

Definition 2.4 (SCS) Let $M$ be the set of sequences $\left\{\widehat{H}^{(k)}\right\}_{k \geq 0}$ of formal Laurent series with coefficients in $\Lambda$ admitting the following expansion in $z$ :

$$
\left\{\begin{array}{l}
\widehat{H}^{(2 k)}=z^{k}+\sum_{j>0}\left(\widehat{H}_{0, j}^{2 k} z^{-j}+\widehat{H}_{1, j}^{2 k} \theta z^{-j}\right) \\
\widehat{H}^{(2 k+1)}=\theta z^{k}+\sum_{j>0}\left(\widehat{H}_{0, j}^{2 k+1} z^{-j}+\widehat{H}_{1, j}^{2 k+1} \theta z^{-j}\right)
\end{array}\right.
$$

\footnotetext{
${ }^{2}$ To make contact with the previous definitions, notice that $V_{B_{[x \varphi]}}=V \otimes_{\Lambda} B_{[x \varphi]}$.
} 
with $\widehat{H}^{(0)}=1, \overline{\widehat{H}^{(k)}}=k \bmod 2$, and let

$$
W=\operatorname{span}_{\Lambda}\left\{\widehat{H}^{(k)}, k \geq 0\right\} \subset V .
$$

It is not difficult to realize that $M$ is isomorphic to the big cell of the $0 \mid 0$ component of $S G r_{\Lambda}$ (i.e. the open subset where $\operatorname{ker} \pi_{W}=\operatorname{coker} \pi_{W}=0$ ), an explicit isomorphism being given by the map

$$
\left\{\widehat{H}^{(k)}\right\}_{k \geq 0} \mapsto \operatorname{span}_{\Lambda}\left\{\widehat{H}^{(k)}, k \geq 0\right\}
$$

The super central system is the dynamical system defined on $M$ by requiring that

$$
\left(\partial_{t_{j}}+\widehat{H}^{(j)}\right) \cdot W \subset W
$$

or, equivalently,

$$
\partial_{t_{j}} \widehat{H}^{(k)}=-\pi_{-}\left(\widehat{H}^{(j)} \widehat{H}^{(k)}\right),
$$

where $\pi_{-}: V \rightarrow V_{-}$is the projection of $V=V_{-} \oplus V_{+}$onto $V_{-}$parallel to $V_{+}$.

By comparing coefficients in the formulation (2.18) of the super central system, we can explicitly write its evolution equations (see Table 2). Notice that the SCS is to be thought of as a system of $\mathbb{Z}_{2}$-graded ordinary differential equations.

\subsection{HSKP as a "reduction" of SCS}

The Hamiltonian super KP hierarchy (2.14) can be obtained from SCS by "spatialisation". This procedure will be used in Section 4 to produce solutions of HSKP starting from solutions of SCS. A spatialization of a hierarchy of dynamical systems $X$ is a process, (see, e.g., $\mathbb{\|}$ ), consisting in the projection of $X$ onto the space $\widehat{\mathcal{Q}}_{j}$ of solutions of its $j$-th flow. More informally, it boils down to interpret a distinguished flow parameter as a space coordinate, and allows to interpret the dynamical system as a system of PDEs.

In the ordinary KP case, spatialization with respect to the time $t_{1}=x$ simply amounts to identify $t_{1}$ with $x$ (or better, substitute $t_{1}=x+t_{1}$ in the solutions of $\mathrm{CS})$. This procedure yields that $h(x)=H_{\left.\right|_{t_{1}=t_{1}+x}}^{(1)}$ is a solution to the KP equations.

In the super case, we want to consider the projection of SCS to the space $\widehat{\mathcal{Q}}_{2}$ of solutions of its second flow i.e. the space of orbits of $\partial_{2}$. Essentially, we have to consider $k=1$ and interpret the first two families of equations of motion reported in Table 2 as recursive definitions of the currents, as differential polynomials (in the space variable $x=t_{2}$ ) of the generators $\widehat{H}^{(1)}$ and $\widehat{H}^{(2)}$. With respect to the bosonic case, there is a subtlety, connected with the relation of the first time $t_{1}$ of SCS with the fermionic partner $\varphi$ of $x$. Observe that, by the definition of the super central 
Table 2: The SCS equations

$$
\begin{aligned}
\partial_{t_{2 k}} \widehat{H}^{(2 j)=} & \widehat{H}^{(2 j+2 k)}-\widehat{H}^{(2 k)} \widehat{H}^{(2 j)}+\sum_{l=1}^{j}\left(\widehat{H}_{0, l}^{2 k} \widehat{H}^{(2 j-2 l)}+\widehat{H}_{1, l}^{2 k} \widehat{H}^{(2 j-2 l+1)}\right) \\
& +\sum_{l=1}^{k}\left(\widehat{H}_{0, l}^{2 j} \widehat{H}^{(2 k-2 l)}+\widehat{H}_{1, l}^{2 j} \widehat{H}^{(2 k-2 l+1)}\right) \\
\partial_{t_{2 k}} \widehat{H}^{(2 j+1)=} & \widehat{H}^{(2 j+2 k+1)}-\widehat{H}^{(2 k)} \widehat{H}^{(2 j+1)}+\sum_{l=1}^{j} \widehat{H}_{0, l}^{2 k} \widehat{H}^{(2 j-2 l+1)} \\
& +\sum_{l=1}^{k}\left(\widehat{H}_{0, l}^{2 j+1} \widehat{H}^{(2 k-2 l)}+\widehat{H}_{1, l}^{2 j+1} \widehat{H}^{(2 k-2 l+1)}\right) \\
\widehat{\partial}_{t_{2 k+1}} \widehat{H}^{(2 j)=} & \widehat{H}^{(2 j+2 k+1)}-\widehat{H}^{(2 k+1)} \widehat{H}^{(2 j)}+\sum_{l=1}^{k} \widehat{H}_{0, l}^{2 j} \widehat{H}^{(2 k-2 l+1)} \\
& +\sum_{l=1}^{j}\left(\widehat{H}_{0, l}^{2 k+1} \widehat{H}^{(2 j-2 l)}+\widehat{H}_{1, l}^{2 k+1} \widehat{H}^{(2 j-2 l+1)}\right) \\
\partial_{t_{2 k+1}} \widehat{H}^{(2 j+1)=} & -\widehat{H}^{(2 k+1)} \widehat{H}^{(2 j+1)}+\sum_{l=1}^{j} \widehat{H}_{0, l}^{2 k+1} \widehat{H}^{(2 j-2 l+1)}-\sum_{l=1}^{k} \widehat{H}_{0, l}^{2 j+1} \widehat{H}^{(2 k-2 l+1)}
\end{aligned}
$$

system, we have $\partial_{t_{j}} \widehat{H}^{(k)}=(-1)^{j k} \partial_{t_{k}} \widehat{H}^{(j)}$. Notice in particular that $\partial_{1} \widehat{H}^{(1)}=0$. Now, for $k>1$

$$
(-1)^{k}\left(\partial_{1}+t_{1} \partial_{2}\right) \widehat{H}^{(k)}=\partial_{t_{k}}\left(\widehat{H}^{(1)}+t_{1} \widehat{H}^{(2)}\right),
$$

suggesting that in order to get HSKP (and solutions thereof) we should put $\hat{h}=$ $\widehat{H}^{(1)}+t_{1} \widehat{H}^{(2)}, t_{2}=x$ and $t_{1}=\varphi$. This is "almost true", but one must pay attention to the order in which these identifications are performed. Indeed, plugging $k=1$ in the left hand side of (2.19), we get

$$
-\left(\partial_{1}+t_{1} \partial_{2}\right) \widehat{H}^{(1)}=-t_{1} \partial_{1} \widehat{H}^{(2)} \neq \partial_{1}\left(\widehat{H}^{(1)}+t_{1} \widehat{H}^{(2)}\right),
$$

which is unconsistent.

The right way to proceed is the following. Starting from a solution of SCS, which depends on the times $\left(t_{1}, t_{2}, \ldots\right)=\mathbf{t}$, one first replaces in the currents $\widehat{H}^{(j)}(\mathbf{t})$ the times $t_{1}$ with $t_{1}+\varphi$ and $t_{2}$ with $x$, then one defines

$$
\hat{h}(x, \varphi ; \mathbf{t}):=\widehat{H}^{(1)}\left(t_{1}+\varphi, x, \ldots\right)+\varphi \widehat{H}^{(2)}\left(t_{1}+\varphi, x, \ldots\right) .
$$


Since $\partial_{1} \widehat{H}^{(k)}=\partial_{\varphi} \widehat{H}^{(k)}$ for any $k$, and taking also into account $(2.19)$, now we have that the field $\hat{h}(x, \varphi ; \mathbf{t})$ is a solution of $\partial_{t_{k}} \hat{h}(x, \varphi ; \mathbf{t})=(-1)^{k} \delta \widehat{H}^{(k)}$, i.e. that it indeed satisfies the HSKP hierarchy. Observe that $\widehat{H}^{(2)}=\hat{h}^{(2)}=(\delta \hat{h})$; in fact

$$
\begin{aligned}
\hat{h}^{(2)} & =\delta\left(\widehat{H}^{(1)}+\varphi \widehat{H}^{(2)}\right)=\partial_{\varphi} \widehat{H}^{(1)}+\varphi \partial_{x} \widehat{H}^{(1)}+\partial_{\varphi}\left(\varphi \widehat{H}^{(2)}\right) \\
& =\varphi \partial_{\varphi} \widehat{H}^{(2)}+\left(\widehat{H}^{(2)}-\varphi \partial_{\varphi} \widehat{H}^{(2)}\right)=\widehat{H}^{(2)}
\end{aligned}
$$

Finally one has that $\left(\partial_{t_{j}}+\widehat{H}^{(j)}\right) W \subset W$ implies $(\delta+\hat{h}) W_{B_{[x \varphi]}} \subset W_{B_{[x \varphi]}}$.

\subsection{The connection with the JSKP of Mulase and Rabin}

In this section we will show that HSKP is equivalent to the Mulase-Rabin Jacobian Super KP hierarchy. Although it seems conceivable from the supercommutativity of the flows, such an identification is somewhat subtle. Our essential tool will be the introduction of two wave or Baker-Akhiezer functions associated with HSKP.

The zero curvature condition (2.16) implies the existence of a first wave function $\Phi$ satisfying

$$
\frac{\partial}{\partial t_{k}} \Phi=\widehat{H}^{(k)} \Phi
$$

Now we perform the following "trick", whose meaning will be discussed in Remark 2.1. We define "enhanced" currents $\widehat{\mathcal{H}}^{(j)}$ by the formula:

$$
\widehat{\mathcal{H}}^{(j)}=\widehat{H}^{(j)}+(-1)^{j+1} \varphi \oint \widehat{H}^{(j)} d z d \theta
$$

In words, the difference between $\widehat{\mathcal{H}}^{(j)}$ and $\widehat{H}^{(j)}$ is (up to a sign) the $\theta$ component of the residue in $z$ of $\widehat{H}^{(j)}$, multiplied by $\varphi$; in the sequel we will denote it as

$$
\widehat{\mathcal{H}}^{(j)}-\widehat{H}^{(j)}=(-1)^{j+1} \varphi C_{j} .
$$

The zero curvature condition on the currents $\widehat{H}^{(j)}$ implies that the enhanced currents satisfy the analogue condition

$$
\frac{\partial \widehat{\mathcal{H}}^{(j)}}{\partial t_{n}}=(-1)^{j n} \frac{\partial \widehat{\mathcal{H}}^{(n)}}{\partial t_{j}},
$$

and so guarantees the existence of an enhanced wave function $\Psi$ satisfying

$$
\frac{\partial}{\partial t_{n}} \Psi=\widehat{\mathcal{H}}^{(n)} \Psi
$$

The wave function $\Psi$ is readily seen to be related to the $\Phi$-wave function by the formula:

$$
\Psi=\Phi \cdot \exp \left(-\varphi \int_{\mathbf{t}_{0}^{e v}}^{\mathbf{t}^{e v}} \sum_{n} C_{2 n} d s_{2 n}\right) .
$$


We now consider the logarithmic derivative

$$
\hat{\mathfrak{h}}=\delta \Psi / \Psi .
$$

It is related with the Faà di Bruno generator $\hat{h}$ by

$$
\hat{\mathfrak{h}}=\hat{h}-\int_{\mathbf{t}_{0}^{e v}}^{\mathbf{t}^{e v}} \sum_{n} C_{2 n} d s_{2 n} .
$$

Actually, since $C_{2}$ is readily seen to be $\psi_{1}$, we can say that $\hat{\mathfrak{h}}$ is a $\mathbb{C}^{*}[z, \theta]$-valued superfield of the form

$$
\hat{\mathfrak{h}}=\theta a+\tilde{\nu}+\varphi h+(\theta \varphi) \psi,
$$

where now

$$
\tilde{\nu}=\nu_{0}+\sum_{j \geq 1} \frac{\nu_{j}}{z^{j}}, \quad \text { with } \nu_{0 x}=-\psi_{1} .
$$

We notice that, by a straightforward supersymmetric extension of standard properties of the Faà di Bruno procedure, since $\hat{\mathfrak{h}}$ differs from $\hat{h}$ by a zero order term in $z$, one can write the Faà di Bruno iterates $\hat{\mathfrak{h}}^{(j)}$ of $\hat{\mathfrak{h}}$ as a linear (over $B_{[x \varphi]}$ ) combination of the iterates $\hat{h}^{(j)}$ we have been using so far (and conversely). To grasp this fact, one simply has to notice that Lemma 2.1 holds irrespectively of the fact that $\nu_{0}$ vanishes. Since $\hat{f}^{\prime}=\delta \hat{\mathfrak{h}}=\hat{f}+\delta \nu_{0}$, we have (using induction) that

$$
\begin{aligned}
\left(\partial_{x}+\hat{f}^{\prime}\right) & \sum \alpha_{i} \hat{h}^{(i)}=\left(\partial_{x}+\hat{f}+\delta \nu_{0}\right)\left(\sum \alpha_{i} \hat{h}^{(i)}\right) \\
& =\sum \alpha_{i} \hat{h}^{(i+2)}+\sum \alpha_{i x} \hat{h}^{(i)}+\sum\left(\delta \nu_{0} \alpha_{i}\right) \hat{h}^{(i)} .
\end{aligned}
$$

Summing up, we see that we can express the enhanced currents as linear combinations of the Faà di Bruno iterates of $\hat{\mathfrak{h}}$ :

$$
\widehat{\mathcal{H}}^{(j)}=\sum_{m} \Gamma_{m}^{j} \hat{\mathfrak{h}}^{(m)} .
$$

Thanks to the obvious equality $\hat{\mathfrak{h}}^{(j)} \Psi=\delta^{j} \Psi$, we can write equation (2.24) as follows:

$$
\partial_{t_{j}} \Psi=\widehat{\mathcal{H}}^{(j)} \Psi=\sum_{l} \Gamma_{l}^{j} \hat{\mathfrak{h}}^{(l)} \Psi=B_{j} \cdot \Psi,
$$

where $B_{j}$ is a super differential operator of order $j$ and of parity $j$ mod 2 .

This equation is the bridge between HSKP and JSKP. The latter is usually formulated within the theory of super pseudo-differential operators. A discussion of such a topic is outside the size of this paper (see, e.g., 25, 29, 30 for details). We need the following lemma, whose proof is a straightforward computation: 
Lemma 2.3 Let $S$ be a super pseudo-differential operator, with coefficients in $B_{[x \varphi]}$ of the form

$$
S=1+\sum_{j>0}\left(u_{j}+\varphi \xi_{j}\right) \partial_{x}^{-j}+\left(\eta_{j}+\varphi w_{j}\right) \delta^{-(2 j-1)}
$$

and let

$$
e(z, \theta ; x, \varphi, \mathbf{t})=\exp \left(\varphi \theta+z x+\sum_{j>0}\left(t_{2 j} z^{j}+t_{2 j-1} \theta z^{j-1}\right)\right)
$$

be the vacuum wave function (in the terminology of [19]) for JSKP. Then, if $\Psi$ is a Baker-Akhiezer function for JSKP obtained by dressing with $S$ the vacuum wave function $e$,

$$
\Psi=S \cdot e
$$

its logarithmic derivative $\delta \Psi / \Psi$ is a superfield of the form (2.28), satisfying the constraint $\nu_{0 x}+\psi_{1}=0$.

The JSKP equations can now be obtained by means of standard procedures in the theory of integrable systems. Indeed, taking the $t_{2 j}$ and $t_{2 j-1}$ derivatives of the dressing relation (2.32), and taking also equation (2.30) into account, we have

$$
\begin{aligned}
\partial_{t_{2 j}} \Psi & =\partial_{t_{2 j}}(S \cdot e)=\left(\partial_{t_{2 j}} S\right) \cdot e+S \cdot z^{j} e=\left(\partial_{t_{2 j}} S\right) \cdot e+S \delta^{2 j} \cdot e \\
& =\left(\left(\partial_{t_{2 j}} S\right) S^{-1}+S \delta^{2 j} S^{-1}\right) S \cdot e=\left(\left(\partial_{t_{2 j}} S\right) S^{-1}+S \delta^{2 j} S^{-1}\right) \cdot \Psi=B_{2 j} \cdot \Psi
\end{aligned}
$$

and

$$
\begin{aligned}
\partial_{t_{2 j-1}} \Psi & =\partial_{t_{2 j-1}}(S \cdot e)=\left(\partial_{t_{2 j-1}} S\right) \cdot e+S \cdot \theta z^{j-1} e=\left(\partial_{t_{2 j-1}} S\right) \cdot e+S\left(\delta^{2 j-1}-\varphi \delta^{2 j}\right) \cdot e \\
& =\left(\left(\partial_{t_{2 j-1}} S\right) S^{-1}+S\left(\delta^{2 j-1}-\varphi \delta^{2 j}\right) S^{-1}\right) \cdot \Psi=B_{2 j-1} \cdot \Psi
\end{aligned}
$$

Since $\left(\partial_{j} S\right) S^{-1}=\left(\left(\partial_{j} S\right) S^{-1}\right)_{-}$is a purely pseudo-differential operator (i.e. it has no differential part) we get

$$
\partial_{t_{2 j}} S=-\left(S \delta^{2 j} S^{-1}\right)_{-} S=-\left(S \partial_{x}^{j} S^{-1}\right)_{-} S
$$

and

$$
\partial_{t_{2 j-1}} S=-\left(S\left(\delta^{2 j-1}-\varphi \delta^{2 j}\right) S^{-1}\right)_{-} S=-\left(S \partial_{\varphi} \partial_{x}^{j-1} S^{-1}\right)_{-} S,
$$

which are the equations that Mulase and Rabin defined for JSKP.

Remark 2.1 As we have anticipated, the introduction of the enhanced currents $\widehat{\mathcal{H}}^{(j)}$ is not a mere trick. To better understand their origin it is useful to reconsider our choices, namely the decomposition (2.17) of the space $V_{B_{[x \varphi]}}$ of formal Laurent series with coefficients in $B_{[x \varphi]}$ as the direct sum of the subspace generated by the Faà di Bruno monomials $W_{B_{[x \varphi]}}$ and the space $V_{B_{[x \varphi]}}^{-}$of formal power series without "constant 
term", i.e. starting from $z^{-1}$ and $\theta z^{-1}$. If $\pi_{+}: V_{B_{[x \varphi]}} \rightarrow W_{B_{[x \varphi]}}$ is the projection associated with such a decomposition, then the currents $\widehat{H}^{(k)}$ are given by the formulas

$$
\widehat{H}^{(2 j+p)}=\pi_{+}\left(z^{j} \theta^{p}\right), j \in \mathbb{N}, p \in\{0,1\} .
$$

Actually, associated with our geometrical datum, there is another natural choice. Indeed, one simply notice the fact that it is possible to extend the Faà di Bruno recursion relations (2.8) to negative values of the index $j$, and get a full Faà di Bruno basis $\left\{\hat{\mathfrak{h}}^{(j)}\right\}_{j \in \mathbb{Z}}$ in $V_{B_{[x \varphi]}}$. The asymptotics of the Faà di Bruno basis is readily seen to be

$$
\hat{\mathfrak{h}}^{(2 j)} \sim z^{j}, \quad \hat{\mathfrak{h}}^{(2 j-1)} \sim \theta z^{j-1}+\varphi z^{j}
$$

Hence we have another natural decomposition

$$
V_{B_{[x \varphi]}}=W_{B_{[x \varphi]}} \oplus W_{B_{[x \varphi]}^{-}}^{-}
$$

where now $W_{B_{[x \varphi]}}^{-}$is the space generated by the Faà di Bruno iterates with negative index. If we call

$$
\pi_{+}^{\prime}: V_{B_{[x \varphi]}} \rightarrow W_{B_{[x \varphi]}}
$$

the projection associated with this new decomposition, then we have that the enhanced currents are given by

$$
\widehat{\mathcal{H}}^{(2 j+p)}=\pi_{+}^{\prime}\left(z^{j} \theta^{p}\right), j \in \mathbb{N}, p \in\{0,1\} .
$$

Actually, it is not surprising that the connection with the usual formulation of the theory by means of super pseudo-differential operators can be better described using the decomposition associated with the full Faà di Bruno basis, since $\delta^{j} \Psi=\hat{\mathfrak{h}}^{(j)} \Psi$ and the projection $\pi_{+}^{\prime}$ is adapted to the projection which kills the non-differential part of a super pseudodifferential operator.

Remark 2.2 It is actually possible to write an evolution equation of the form (2.30) for the first wave function $\Phi$ as well. Indeed, since $\hat{h} \Phi=\delta \Phi$ and $\widehat{H}^{(j)}$ is a linear combination of the Faà di Bruno iterates $\hat{h}^{(j)}$ of $\hat{h}$, we can read the equation $\partial_{t_{j}} \Phi=$ $\widehat{H}^{(j)} \Phi$ as $\partial_{t_{j}} \Phi=B_{j}^{\prime} \Phi$ where $B_{j}^{\prime}$ is still a super differential operator of order $j$ and of parity $j \bmod 2$. However, we can no more express $\Phi$ as the result of the action of a generic dressing operator $S$ on the vacuum wave function $e$ of Lemma 2.3. Moreover, the HSKP equations are not compatible with $\Phi$ having such an expression: even if $\psi_{1}=0$ at $\mathbf{t}_{0}$, this is no more true for the evolved field so $0=\nu_{0 x} \neq-\psi_{1}$.

Remark 2.3 As we have seen, the connection between HSKP and JSKP is (albeit in a tricky way) a change of coordinates. The relation among the degrees of 
freedom $\left(u_{i}, w_{i}, \xi_{i}, \eta_{i}\right)$ of $S$ and the degrees of freedom $\left(a_{i}, h_{i}, \nu_{i}, \psi_{i}\right)$ (collected in $(a(z), h(z), \nu(z) \psi(z))$ as usual) of $\hat{\mathfrak{h}}$ is indeed the following:

$$
\begin{aligned}
& \nu(z)\left(1+\sum_{i>1} \frac{u_{i}}{z^{i}}\right)=-\eta_{1}+\sum_{i>1} \frac{\xi_{i}-\eta_{i+1}}{z^{i}} ; \\
& a(z)\left(1+\sum_{i>1} \frac{u_{i}-\eta_{i} \nu(z)}{z^{i}}\right)=1+\sum_{i>1} \frac{u_{i}+w_{i}}{z^{i}} ; \\
& h(z)\left(1+\sum_{i>1} \frac{u_{i}}{z^{i}}\right)=z+u_{1}+\eta_{1} \nu(z)+\sum_{i>1} \frac{u_{i, x}+u_{i+1}}{z^{i}} \\
& +\nu(z)\left(\sum_{i>1} \frac{\xi_{i}-\eta_{i+1}}{z^{i}}\right) ; \\
& \psi(z)\left(1+\sum_{i>1} \frac{u_{i}}{z^{i}}\right)=a(z)\left(\eta_{1}+\sum_{i>1} \frac{\eta_{i+1}-\xi_{i}}{z^{i}}\right)+\nu(z) \sum_{i>1} \frac{w_{i}}{z^{i}} \\
& -h(z) \sum_{i>1} \frac{\eta_{i}}{z^{i}}+\sum_{i>1} \frac{\eta_{i x}+\xi_{i}}{z^{i}} .
\end{aligned}
$$

These equations give $\left(a_{i}, h_{i}, \nu_{i}, \psi_{i}\right)$ as differential polynomials in the $\left(u_{i}, w_{i}, \xi_{i}, \eta_{i}\right)^{\prime}$ 's, and can be inverted modulo quadratures (as usual in the theory of KP-like equations). As we shall show in the next sections, there is some merit in considering such non standard coordinates, whose choice is suggested by the supersymmetric extension of Gel'fand-Zakharevich set-up for the KP theory.

\subsection{A super $\mathrm{KdV}$ equation as a reduction of HSKP}

We will discuss now a supersymmetric generalization of the KdV equation obtained as reduction of the Hamiltonian super KP hierarchy. This example will be important in giving us one more clue to the second part of the paper. It can be shown that constraints of the form

$$
\widehat{H}^{(2 k)}=z^{k}
$$

are compatible with HSKP. We consider $k=2$ obtaining

$$
\begin{aligned}
& \left\{\begin{array}{l}
h_{2}=-\frac{1}{2} h_{1}^{\prime}+\psi_{1} \nu_{1} \\
h_{k}=-\frac{1}{2} h_{k-1}^{\prime}-\frac{1}{2} \sum_{j=1}^{k-2} h_{k-j-1} h_{j}+\psi_{1} \nu_{k-1} \quad \text { for } k>2,
\end{array}\right. \\
& \left\{\begin{array}{l}
\psi_{2}=-\frac{1}{2} \psi_{1}^{\prime}+a_{1} \psi_{1} \\
\psi_{k}=-\frac{1}{2} \psi_{k-1}^{\prime}-\sum_{j=1}^{k-2} h_{k-j-1} \psi_{j}+a_{k-1} \psi_{1} \quad \text { for } k>2,
\end{array}\right. \\
& \left\{\begin{array}{l}
\nu_{2}^{\prime}=-\frac{1}{2} \nu_{1}^{\prime \prime}+a_{1}^{\prime} \nu_{1} \\
\nu_{k}^{\prime}=-\frac{1}{2} \nu_{k-1}^{\prime \prime}-\sum_{j=1}^{k-2} h_{k-j-1} \nu_{j}^{\prime}+a_{1}^{\prime} \nu_{k-1} \quad \text { for } k>2,
\end{array}\right. \\
& \left\{\begin{array}{l}
a_{2}^{\prime}=-\frac{1}{2} a_{1}^{\prime \prime}+a_{1} a_{1}^{\prime} \\
a_{k}^{\prime}=-\frac{1}{2} a_{k-1}^{\prime \prime}-\sum_{j=1}^{k-2} h_{k-j-1} a_{j}^{\prime}+a_{k-1} a_{1}^{\prime} \quad \text { for } k>2 .
\end{array}\right.
\end{aligned}
$$

These equations allow us to compute recursively the coefficients $h_{j}, \psi_{j}, \nu_{j}$ and $a_{j}$ for $j>1$ in terms of $h_{1}, \psi_{1}, \nu_{1}$ and $a_{1}$ by means of quadratures. In order to explicitly write the equations for the independent degrees of freedom we have only to calculate 
the coefficients of order 1 of the super current densities. Since the relations (2.38) algebraically determine only the derivatives of the fields $a_{i}, \nu_{i}, 1 \geq 2$, in general the resulting equations will be integro-differential ones. Fortunately enough, for the sixth time of the hierarchy the non local terms cancel each other, and the result is

$$
\left\{\begin{array}{l}
\partial_{6} \nu_{1}=\frac{1}{4} \nu_{1}^{\prime \prime \prime}-\frac{3}{2} a_{1}^{\prime} \nu_{1}^{\prime}-3 h_{1} \nu_{1}^{\prime} \\
\partial_{6} a_{1}=\frac{1}{4} a_{1}^{\prime \prime \prime}-\frac{3}{2} a_{1}^{\prime 2}-3 h_{1} a_{1}^{\prime}+6 \psi_{1} \nu_{1}^{\prime} \\
\partial_{6} h_{1}=\frac{1}{4} h_{1}^{\prime \prime \prime}-3 h_{1} h_{1}^{\prime}-\frac{3}{2} \psi_{1} \nu_{1}^{\prime \prime}-\frac{3}{2} \psi_{1}^{\prime} \nu_{1}^{\prime} \\
\partial_{6} \psi_{1}=\frac{1}{4} \psi_{1}^{\prime \prime \prime}-\frac{3}{2} a_{1}^{\prime \prime} \psi_{1}-\frac{3}{2} a_{1}^{\prime} \psi_{1}^{\prime}-3 h_{1} \psi_{1}^{\prime}-3 h_{1}^{\prime} \psi_{1}
\end{array} .\right.
$$

We thus see that the evolution equations for the time $t_{6}$ are a supersymmetric extension of the $\mathrm{KdV}$ equation, which can be retrieved by setting $a_{1}=\nu_{1}=\psi_{1}=0, h_{1}=\frac{u}{2}$.

We notice also the following fact. Substituting $\nu=\psi=0, h=\frac{u}{2}$ in the above equations (2.39) we obtain the ordinary system of PDE's in two variables $u$ and $a$

$$
\left\{\begin{array}{l}
\partial_{t} a=\frac{1}{4} a_{x x x}-\frac{3}{2} a_{x}{ }^{2}-\frac{3}{2} u a_{x} \\
\partial_{t} u=\frac{1}{4} u_{x x x}-\frac{3}{2} u u_{x}
\end{array} .\right.
$$

One can easily notice that the submanifold defined by $u=-a_{x}+a^{2}+\lambda_{0}$ is an invariant submanifold of these equations, where the first one coincides with the modified $\mathrm{KdV}$ equation. So we see that this reduction of HSKP "contains" both KdV and mKdV. This observation will be formalized and explained in the next sections.

\section{HSKP and Darboux transformations}

In general, a Darboux transformation is a way to connect two systems of differential equations enabling to produce a solution of the second once a solution of the first has been supplied. This technique has proved to be very effective both in the construction of large classes of explicit solutions of soliton equations and in the understanding of the nature of infinite dimensional integrable systems (see, e.g., 27, 15]). An example of such transformation is provided by the Miura map in the KdV theory and the modified KdV hierarchy (mKdV) (see, e.g., [8, 27] and the references quoted therein). Here we are mostly interested in the concept of Darboux intertwiners and Darboux coverings introduced in [22, 23], where the geometrical features of the method where analyzed as follows.

Consider three vector fields $X, Y$ and $Z$ on three manifolds $M, N$ and $P$, respectively.

Definition 3.1 29] The vector field $Y$ intertwines $X$ and $Z$ if there exists a pair of maps $(\mu: N \rightarrow M, \sigma: N \rightarrow P)$ such that $X=\mu_{*} Y$ and $Z=\sigma_{*} Y$. Moreover, if $X=Z, N$ is a fiber bundle on $M=P$, and $\mu: N \rightarrow M$ is the bundle projection, then $Y$ is said to be a Darboux covering of $X$, and the map $\sigma$ the associated Miura map. Finally, still when $X=Z$ and $\mu: N \rightarrow M$ is a fibre bundle, for each section $\rho: M \rightarrow N$ of $\mu$ which is invariant under $Y$, the composition $\eta=\sigma \circ \rho$ which sends $X$ in $X$, and hence produces an integral curve $\tilde{x}(t)$ of $X$ from the integral curve $x(t)$ of $X$, is called a Darboux transformation. 
Figure 1: The Darboux Maps

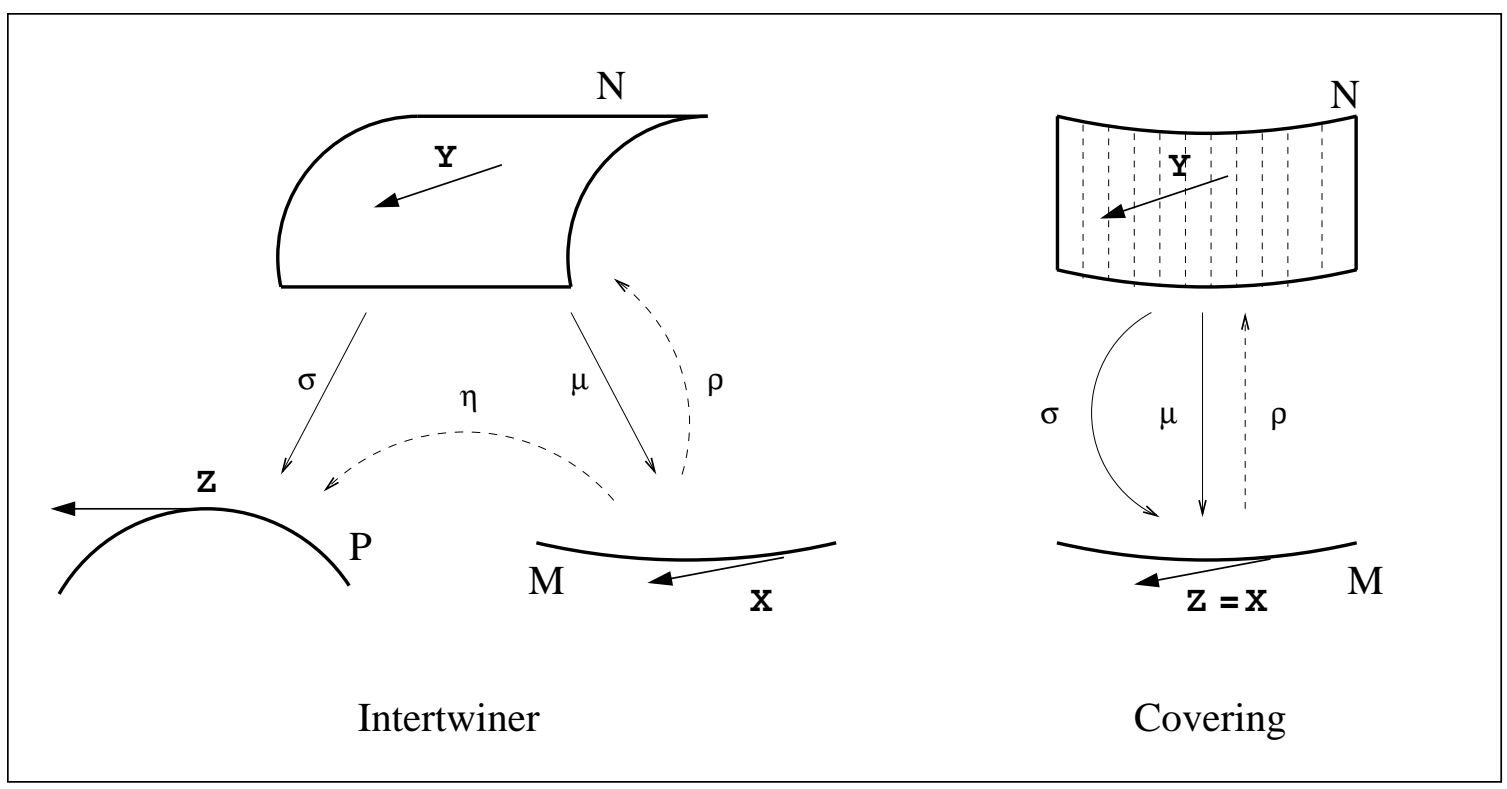

The concept of Darboux covering is useful for constructing both solutions and invariant submanifolds of the vector field $X$ : if $U$ is a chart on $M$ with coordinate $x$ and $V \subset \mu^{-1}(U)$ a chart on $N$ adapted to the projection $\mu$ and with fibered coordinates $(x, a)$, then the local expression of the above vector fields is

$$
\begin{aligned}
& \dot{x}=X(x) \\
& \dot{a}=Y(x, a),
\end{aligned}
$$

where the first equation is that of $X$ on $U$. Then, any integral curve $x(t)$ of $X$ can be lifted to an integral curve $(x(t), a(t))$ of $Y$ by solving the second equation, which is controlled by $x(t)$. Therefore, we get a new integral curve of $X$ by setting

$$
\tilde{x}(t)=\sigma(x(t), a(t)) .
$$

The last equation can also be interpreted as a "symmetry (or Darboux) transformation" of the dynamical system described by $X$, depending on a solution of the auxiliary system for $a$, controlled by $X$ itself, which associates $\tilde{x}(t)$ with $x(t)$.

The application of the formalism we have just described to KP naturally leads to the DKP hierarchy.

Definition 3.2 Let $M$ be the affine space of (formal) monic Laurent series in $z^{-1}$ with coefficients in $C^{\infty}\left(S^{1}\right)$ and of the form

$$
h(z, x)=z+\sum_{j>0} h_{j}(x) z^{-j}
$$


and let $N$ be the affine space of couples $(h, a)$ where $h$ is as above and a is a monic Laurent series of the form

$$
a(z, x)=z+\sum_{j \geq 0} a_{j}(x) z^{-j}
$$

Define two maps $\mu, \sigma: N \rightarrow M$ by

$$
\mu(h, a)=h
$$

and

$$
\tilde{h}:=\sigma(h, a)=h+\frac{\partial_{x} a}{a} .
$$

Finally, let $H^{(k)}$ and $\tilde{H}^{(k)}$ be the current densities associated with $h$ and $\tilde{h}$, respectively. The DKP hierarchy is the hierarchy of evolution equations on $N$ defined by

$$
\left\{\begin{array}{rl}
\frac{\partial}{\partial t_{k}} h & =\partial_{x} H^{(k)} \\
\frac{\partial}{\partial t_{k}} a & =a\left(\tilde{H}^{(k)}-H^{(k)}\right)
\end{array} .\right.
$$

DKP is a Darboux covering, in the sense of Definition 3.1, of the KP hierarchy

$$
\partial_{t_{k}} h=\partial_{x} H^{(k)}
$$

Indeed, it is clear that $\mu_{*}$ maps the vector fields $\partial_{t_{j}}$ of DKP to those of KP. As for $\sigma_{*}$, we have

$$
\begin{aligned}
\partial_{t_{k}}\left(\frac{\partial_{x} a}{a}\right) & =\frac{a \partial_{x} \partial_{t_{k}} a-\left(\partial_{x} a\right)\left(\partial_{t_{k}} a\right)}{a^{2}} \\
= & \frac{a \partial_{x}\left(a \tilde{H}^{(k)}-a H^{(k)}\right)-a\left(\partial_{x} a\right)\left(\tilde{H}^{(k)}-H^{(k)}\right)}{a^{2}} \\
= & \partial_{x} \tilde{H}^{(k)}-\partial_{x} H^{(k)}
\end{aligned}
$$

and finally

$$
\partial_{t_{k}} \tilde{h}=\partial_{t_{k}} h+\partial_{t_{k}}\left(\frac{\partial_{x} a}{a}\right)=\partial_{x} \tilde{H}^{(k)} .
$$

In the papers [23, 22, 5, 10], the following results were obtained:

1. The modified KP hierarchy of $\llbracket 18$ is the restriction of DKP on the invariant submanifold $S_{0} \subset N$ defined by the simple equation $a=h+a_{0}$;

2. The DKP equations admit a remarkable family of invariant submanifolds, $S_{l}$, of which $S_{0}$ is the simplest; the images through $\mu$ of the intersections of two (or more) submanifolds, $S_{l_{1}} \cap \cdots \cap S_{l_{k}}$ is an invariant submanifold of KP, which coincide with the rational KP reductions of Dickey and Krichever (see, e.g., [9, 16, 1, 3]); 
3. The central system CS can be explicitly linearized and classes of solutions can be explicitly found by means of a Darboux intertwiner linking it with the Sato system, that is, the coordinate expression of the linear flows of KP on the Sato Grassmannian.

In the rest of this Section we will first show that HSKP (and hence JSKP) can be seen as a supersymmetric extension of DKP; then we will define a Darboux covering for HSKP, and briefly discuss the analogue of the invariant submanifolds mentioned in points 1 and 2 of the above list. The generalization of point 3 will be the subject of Section 1 .

\subsection{HSKP and Darboux transformations}

Our first goal is to give a connection between the Jacobian super KP hierarchy and DKP. First of all, we observe that the role $a$ has in DKP does not depend on the order of its pole, since it appears in a homogeneous way in all the equations. Hence, we see that (the reduction modulo nilpotents elements in $\Lambda$ of) the bosonic degrees of freedom of HSKP are exactly the degrees of freedom of $D K P$ : the Laurent series a appearing in the definition (2.7) of the super Faà di Bruno generator can be identified with $z^{-1}$ times the Laurent series $a$ appearing in the definition 3.2 of DKP. It is thus tempting to conjecture a relation between the two hierarchies. In fact, we can prove the following

Proposition 3.1 Let $\hat{h}$ and $\widehat{H}^{(k)}$ be, respectively, the super Fà̀ di Bruno generator and the currents of HSKP defined as in Section 2.1.

i. The constraint $\nu=\psi=0$ is compatible with the even flows of the HSKP hierarchy.

ii. The reduction $H S K P_{\text {bos }}$ of the even flows of $H S K P$ given by setting $\nu=\psi=0$ is isomorphic to DKP, i.e. if $\hat{h}$ is a solution of $H S K P_{b o s}$, then $(h, z a)$ is a solution of $D K P$ and vice versa.

\section{Proof.}

i. Looking at the recurrence relations we introduced in Section 2.1, namely equations (2.9) and Table 1, we see that, under the constraint $\nu=\psi=0$, one has

$$
\left\{\begin{array}{l}
\hat{h}^{(2 k-1)}=\theta a^{(k)}+\varphi h^{(k)} \\
\hat{h}^{(2 k)}=h^{(k)}-(\theta \varphi) b^{(k)},
\end{array}\right.
$$

where the coefficients are given by the following recursion relations:

$$
\begin{aligned}
& \left\{\begin{array}{l}
h^{(k+1)}=\left(\partial_{x}+h\right) h^{(k)} \\
h^{(0)}=1
\end{array}, \quad\left\{\begin{array}{l}
a^{(k+1)}=\left(\partial_{x}+h\right) a^{(k)} \\
a^{(1)}=a
\end{array},\right.\right. \\
& \left\{\begin{array}{l}
b^{(k+1)}=\left(\partial_{x}+h\right) b^{(k)}+\left(\partial_{x} a\right) h^{(k)} \\
b^{(0)}=0 .
\end{array}\right.
\end{aligned}
$$


In particular $\hat{h}^{(0)}=1, \hat{h}^{(1)}=\theta a+\varphi h, \hat{h}^{(2)}=h-\theta \varphi a_{x}$. This implies that

$$
\widehat{H}^{(2 k)}=H^{(k)}-(\theta \varphi) K^{(k)},
$$

where $H^{(k)}$ is the $k$-th current density of $\mathrm{KP}$ and $K^{(k)}$ is some power series in $z^{-1}$ and $x$ of the form

$$
K^{(k)}(z, x)=\sum_{j>0} K_{j}^{k}(x) z^{-j} .
$$

The evolution equations for the even flows of HSKP are then

$$
\left\{\begin{array}{l}
\partial_{t_{2 k}} \nu=0 \\
\partial_{t_{2 k}} \psi=0 \\
\partial_{t_{2 k}} a=K^{(k)} \\
\partial_{t_{2 k}} h=\partial_{x} H^{(k)}
\end{array}\right.
$$

showing that the constraint $\nu=\psi=0$ is compatible with them.

ii. In the proof of i. we have established also that $h$ evolves according to KP. We need only to understand better the evolution of $a$. We have to show that $K^{(k)} / a+H^{(k)}$ is the $k$-th current density of KP associated with

$$
\widetilde{h}=h+\frac{\partial_{x} a}{a} .
$$

To achieve this we consider the function

$$
\widehat{A}=\left(-\frac{1}{a}+\theta \varphi\right),
$$

and perform the "gauge transformation"

$$
\hat{h}^{(k)} \mapsto \hat{l}^{(k)}:=\widehat{A} \hat{h}^{(k)},
$$

that is, we consider the new vector space $\widetilde{W}=\widehat{A} \cdot W$ generated by the $\hat{l}^{(k)}$ 's. We remark that the first transformed basis elements are:

$$
\begin{aligned}
& \hat{l}^{(0)}=-\frac{1}{a}+(\theta \varphi) \cdot 1, \quad \hat{l}^{(1)}=-\theta-\varphi \frac{h}{a} \\
& \hat{l}^{(2)}=-\frac{h}{a}+(\theta \varphi) \cdot\left(h+\frac{\partial_{x} a}{a}\right)=-\frac{h}{a}+(\theta \varphi) \cdot \widetilde{h} .
\end{aligned}
$$

Observe that since $W$ is generated by the action of the operator $\partial_{x}+\hat{h}^{(2)}$ on the pair $\left(\hat{h}^{(0)}=1, \hat{h}^{(1)}=\theta a+\varphi h\right), \widetilde{W}$ will be generated by the action of $\widehat{A}\left(\partial_{x}+\hat{h}^{(2)}\right) \widehat{A}^{-1}$ on the pair $\left(\hat{l}^{(0)}, \hat{l}^{(1)}\right)$. We notice that

$$
\widehat{A}\left(\partial_{x}+\hat{h}^{(2)}\right) \widehat{A}^{-1}=\partial_{x}+h+\frac{\partial_{x} a}{a}=\partial_{x}+\widetilde{h},
$$


which shows that

$$
\left\{\begin{array}{l}
\hat{l}^{(2 k)}=f(k)+\theta \varphi \widetilde{h}^{(k)} \\
\hat{l}^{(2 k+1)}=-\theta \widetilde{h}^{(k)}+\varphi g^{(k)},
\end{array}\right.
$$

Now we consider the transformed current $\widehat{L}^{(2 k)}=\widehat{A} \widehat{H}^{(2 k)}$. Its $\theta \varphi$ component is clearly given by the sum

$$
\widehat{L}_{\theta \varphi}^{(2 k)}=H^{(k)}+K^{(k)} / a
$$

Since $\widehat{L}^{(2 k)}$ is a finite linear combination of the basis elements $\hat{l}^{(j)}$ it follows that $\widehat{L}_{\theta \varphi}^{(2 k)}$ is the unique combination of the $\tilde{h}^{(k)}$ with the asymptotics

$$
L_{\theta \varphi}^{(2 k)}=H^{(k)}+K^{(k)} / a=z^{k}+O(1 / z) .
$$

Hence it must be equal to $\widetilde{H}^{(k)}$, so we get the desired result

$$
K^{(k)}=a\left(H^{(k)}-\tilde{H}^{(k)}\right) .
$$

\subsection{A Darboux covering for HSKP and the super analogue of its rational reductions}

In this section we return to the full supersymmetric picture, define the Darboux transformations and a D-HSKP hierarchy for the Hamiltonian super KP theory, and show how to obtain the super analogue of Dickey's and Krichever's rational reductions of the KP hierarchy.

We observe that given a Laurent series $\hat{h}$ of the usual form of equation (2.7), and a monic even power series

$$
\hat{p}=p+\theta \zeta+\varphi \xi+(\theta \varphi) q,
$$

with $\bar{p}=\bar{q}=0, \bar{\zeta}=\bar{\xi}=1$ and

$$
\left\{\begin{array}{l}
p=1+\sum_{j>0} p_{j} z^{-j} \\
q=\sum_{j>0} q_{j} z^{-j} \\
\zeta=\sum_{j>0} \zeta_{j} z^{-j} \\
\xi=\sum_{j>0} \xi_{j} z^{-j}
\end{array}\right.
$$

the transformed series

$$
\hat{k}=\hat{h}+\frac{\delta \hat{p}}{\hat{p}}
$$

is still of type (2.7). 
Definition 3.3 (D-HSKP) Let $\widehat{N}$ be the affine space of couples of monic formal Laurent series $(\hat{h}, \hat{p})$, let

$$
\hat{k}=\hat{h}+\frac{\delta \hat{p}}{\hat{p}}
$$

and let $\widehat{K}^{(k)}$ be the $k$-th super current density associated to $\hat{k}$. The Darboux-Hamiltonian super KP hierarchy is the set of compatible evolution equations

$$
\left\{\begin{array}{l}
\partial_{t_{k}} \hat{h}=(-1)^{k} \delta \widehat{H}^{(k)} \\
\partial_{t_{k}} \hat{p}=\hat{p}\left(\widehat{K}^{(k)}-\widehat{H}^{(k)}\right)
\end{array} .\right.
$$

If we let $\widehat{M}$ be the affine space of the monic formal Laurent series $\hat{h}$ and we define two maps $\hat{\mu}, \hat{\sigma}: \widehat{N} \rightarrow \widehat{M}$ by

$$
\hat{\mu}(\hat{h}, \hat{p})=\hat{h}
$$

and

$$
\hat{\sigma}(\hat{h}, \hat{p})=\hat{h}+\frac{\delta \hat{p}}{\hat{p}}
$$

then

$$
\begin{aligned}
\partial_{t_{k}}\left(\frac{\delta \hat{p}}{\hat{p}}\right) & =(-1)^{k} \frac{\hat{p} \delta \partial_{t_{k}} \hat{p}-(\delta \hat{p})\left(\partial_{t_{k}} \hat{p}\right)}{\hat{p}^{2}} \\
= & (-1)^{k} \frac{\hat{p} \delta\left(\hat{p} \widehat{K}^{(k)}-\hat{p} \widehat{H}^{(k)}\right)-\hat{p}(\delta \hat{p})\left(\widehat{K}^{(k)}-\widehat{H}^{(k)}\right)}{\hat{p}^{2}} \\
= & (-1)^{k} \delta\left(\widehat{K}^{(k)}-\widehat{H}^{(k)}\right)
\end{aligned}
$$

so

$$
\partial_{t_{k}} \hat{k}=\partial_{t_{k}} \hat{h}+\partial_{t_{k}}\left(\frac{\delta \hat{p}}{\hat{p}}\right)=(-1)^{k} \delta \widehat{K}^{(k)},
$$

i.e. D-HSKP is a Darboux covering of HSKP. In the next section we will use this formalism for a geometrical characterization of the analogue of the rational reductions of the KP hierarchy.

\subsection{Super mKP and Rational Hierarchies}

Proposition 3.2 The submanifold $\widehat{\mathcal{S}}_{l}$ of $\widehat{N}$ (see Definition 3.3) characterized by

$$
z^{l / 2} \hat{p} \in W
$$

for l even, or by

$$
\theta z^{(l-1) / 2} \hat{p} \in W
$$

for $l$ odd, is invariant under the flows of the D-HSKP hierarchy, where we recall that $W=\operatorname{span}_{B_{[x \varphi]}}\left\{\hat{h}^{(j)} \mid j \geq 0\right\}$. Consequently, the submanifold

$$
\widehat{\mathcal{T}}_{l}:=\hat{\mu}\left(\widehat{\mathcal{S}}_{l}\right)
$$

of $\widehat{M}$ is invariant under HSKP. 
Proof. We give the proof only for $l=2 n$ even, the other proof is the same up to some obvious change of signs. The condition $(\hat{h}, \hat{p}) \in \widehat{\mathcal{S}}_{l}$ implies the existence of some coefficients $\alpha_{j}(x, \varphi), j=0, \cdots, l$ such that

$$
z^{n} \hat{p}=\sum_{j=0}^{l} \alpha_{j} \widehat{H}^{(j)}
$$

so we have to show that this expression is invariant under the flows of D-HSKP, i.e.

$$
\partial_{t_{k}}\left(z^{l} \hat{p}-\sum_{j=0}^{l} \alpha_{j} \widehat{H}^{(j)}\right)=0
$$

on $\hat{\mathcal{S}}_{2 n}$. Let $W^{\hat{k}}:=\operatorname{span}_{B_{[x \varphi]}}\left\{\hat{k}^{(j)} \mid j \geq 0\right\}$. By definition we have

$$
\hat{p}(\delta+\hat{k})=(\delta+\hat{h}) \hat{p}
$$

and hence $\hat{p}(\delta+\hat{k})^{j}=(\delta+\hat{h})^{j} \hat{p}$. This implies that $z^{l} \hat{p} W^{\hat{k}} \subset W$, and, therefore, using the D-JSKP equations, $\left(\partial_{t_{k}}+\widehat{H}^{(k)}\right) z^{l} \hat{p}=z^{l} \hat{p} \widehat{K}^{(k)} \in W$, i.e.

$$
z^{l} \partial_{t_{k}} \hat{p}+\sum_{j=0}^{l}(-1)^{j k} \alpha_{j} \widehat{H}^{(k)} \widehat{H}^{(j)} \in W .
$$

Using now the property $\partial_{t_{k}} \widehat{H}^{(j)}+\widehat{H}^{(k)} \widehat{H}^{(j)} \in W$, characteristic of HSKP, and comparing the coefficients of $z^{j}$ and $\theta z^{j}$ in equation (3.5) for $j=0, \cdots, l$, we get

$$
z^{l} \partial_{t_{k}} \hat{p}-\sum_{j=0}^{l}(-1)^{j k} \alpha_{j} \partial_{t_{k}} \widehat{H}^{(j)}=\sum_{j=0}^{l}\left(\partial_{t_{k}} \alpha_{j}\right) \widehat{H}^{(j)},
$$

i.e. (3.6) holds.

As a first application of this result we show how, in such a formalism, we obtain a supersymmetric extension of the modified KP hierarchy. We consider the submanifold $\widehat{\mathcal{S}}_{2}$ defined by $z \hat{p} \in W$. It can be seen that these equations entail the following constraints:

$$
\zeta_{1}=0 ; \quad \int_{S^{1}} \xi_{j} d x=0, j \geq 2 ; \quad q_{1}=0 ; \text { and } \int_{S^{1}} q_{i} d x=0 j \geq 2 .
$$

The bosonic sector of the resulting theory covers the invariant submanifold $S_{0}$ of the DKP equations of [22] defined by $a=h+a_{0}$. There it was proven that $\left.D K P\right|_{S_{0}}$ is another form of the modified KP theory of Kupershmidt [18]. Hence, through this result, we obtain that the restriction $\mathrm{D}-\mathrm{SKP}_{\left.\right|_{\mathcal{S}_{2}}}$ provides a direct supersymmetric extension of mKP.

Finally, following [5], one can define and study the "rational-type reductions" of the HSKP hierarchy as the restriction of the D-HSKP hierarchy to the intersection of suitable of invariant submanifolds. In the next example we will briefly describe the simplest case. 
Example 3.1 Let us consider the triple intersection $\widehat{\mathcal{S}}_{124}$, and its image $\widehat{\mathcal{T}}_{124}$ under $\hat{\mu}$, obtained by requiring that the triple $\left(\theta \hat{p}, z \hat{p}, z^{2} \hat{p}\right)$ lie in $W$. Since $\widehat{H}^{(1)}=\theta a+\nu$ we see that, recalling the form of $\hat{p}=p+\theta \zeta+\varphi \xi+(\theta \varphi) q$, the equation $\theta \hat{p} \in W$ implies

$$
\nu=0, \quad \xi=0, \quad a=p .
$$

Intersecting with $\widehat{\mathcal{S}}_{2}$ we get

$$
h=z p-p_{1}, \quad \psi=-z \zeta+\zeta_{1} p, \quad q=\frac{q_{1} p-p_{x}}{z} .
$$

Finally, requiring $z^{2} \hat{p} \in W$ one sees that it is possible to express all the fields $p_{i}, q_{i}, \zeta_{i}$, (and hence all the currents $\widehat{H}^{(j)}$ ) in terms of the two even fields $r=p_{1}, s=p_{2}$ and the two odd fields $\rho=\zeta_{1}, \sigma=\zeta_{2}$. Indeed the equations to be solved are:

$$
\left\{\begin{array}{l}
z^{2}\left(p^{2}-p\right)+z\left(p_{x}-p_{1} p\right)=p_{1, x}+p_{2} \\
z^{2}(2 p \zeta-\zeta)+z\left(\zeta_{x}-\zeta_{1} p^{2}-p_{1} \zeta\right)=\zeta_{1} p+\zeta_{2}+p_{1} p \zeta \\
q_{1}\left(z\left(p^{2}-p\right)+p_{x}-p_{1} p\right)=z\left(2 p p_{x}-p_{x}\right)+p_{x x}-p_{1 x} p-p_{1} p_{x}
\end{array}\right.
$$

From the first one we get

$$
p_{k+2}=-p_{k+1 x}-\sum_{j=1}^{k} p_{j} p_{k-j+2}, \quad k \geq 1
$$

and from the second a similar formula expressing $\zeta_{j+2}, j \geq 1$ in terms of $\zeta_{1}, \zeta_{2}$ and the $p_{k}$ 's. Plugging the first equation into the third one, we finally get

$$
q_{1}=\frac{d}{d x} \log \left(p_{2}+p_{1 x}\right) .
$$

The resulting equations of motion relative to the time $t_{4}$ are the following:

$$
\left\{\begin{array}{l}
\dot{r}=\left(r_{x}+2 s-r^{2}\right)_{x} \\
\dot{s}=-\left(s_{x}+r s\right)_{x} \\
\dot{\rho}=\rho_{x x}+2 \sigma_{x}-2 r \rho_{x}-2 r_{x} \rho+2\left(\log \left(r_{x}+s\right)\right)_{x}\left(\rho_{x}+\sigma-r \rho\right) \\
\dot{\sigma}=-\sigma_{x x}+2 r \rho_{x x}-2\left(r^{2}+s\right) \rho_{x}-2 r_{x} \sigma+2 r\left(\log \left(r_{x}+s\right)\right)_{x}\left(\rho_{x}+\sigma-r \rho\right) .
\end{array}\right.
$$

We notice that these evolution equations for $r$ and $s$ coincide with those of the realization of the well-known AKNS (or two-boson) hierarchy as a rational reduction of the KP hierarchy [16, 5].

\section{Linearization}

The evolution equations of SCS we have introduced in Section 2.2 are not linear, and not directly linearizable. To obtain their "linearized version", allowing to provide 
explicit solutions, we can exploit Darboux covering techniques as it has been done in 110 for KP. The idea is to find a Darboux covering which intertwines the super Central System SCS defined in Section 2.1 with a new hierarchy whose linearization can be achieved by elementary methods.

To this end, let $\widehat{\mathcal{M}}$ be the space of sequences of Laurent series $\left\{\widehat{Y}^{(k)}\right\}_{k \geq 0}$ of the form

$$
\left\{\begin{array}{l}
\widehat{Y}^{(2 k)}=z^{k}+\sum_{j>0}\left(\widehat{Y}_{0, j}^{2 k} z^{-j}+\widehat{Y}_{1, j}^{2 k} \theta z^{-j}\right) \\
\widehat{Y}^{(2 k+1)}=\theta z^{k}+\sum_{j>0}\left(\widehat{Y}_{0, j}^{2 k+1} z^{-j}+\widehat{Y}_{1, j}^{2 k+1} \theta z^{-j}\right),
\end{array}\right.
$$

where $\overline{\widehat{Y}^{(k)}}=k \bmod 2$. The third manifold $\widehat{\mathcal{P}}$ of Definition 3.1 is just a copy of $\widehat{\mathcal{M}}$ formed by the sequences $\left\{\widehat{H}^{(k)}\right\}_{k \geq 0}$. Finally, the manifold $\widehat{\mathcal{N}}$ is the Cartesian product $\widehat{\mathcal{M}} \times \widehat{\mathcal{G}}$ of $\widehat{\mathcal{M}}$ by the group of even invertible formal power series $\hat{w}$ of the form

$$
\hat{w}=1+\sum_{j>0}\left(\hat{w}_{0, j} z^{-j}+\hat{w}_{1, j} \theta z^{-j}\right) .
$$

The next step is to define suitable vector fields $\widehat{\mathcal{X}}, \widehat{\mathcal{Y}}$ and $\widehat{\mathcal{Z}}$ on $\widehat{\mathcal{M}}, \widehat{\mathcal{N}}$ and $\widehat{\mathcal{P}}$, respectively. The vector field $\widehat{\mathcal{Z}}$ is any vector field of SCS, which is completely characterized by

$$
\left(\partial_{t_{k}}+\widehat{H}^{(k)}\right) \cdot W \subset W
$$

The flow can be identified by using an index, so we call this vector field $\widehat{\mathcal{Z}}_{k}$. To define $\widehat{\mathcal{X}}$ we introduce the subspace $W^{(\widehat{Y})}$ of $V$ spanned by the $\widehat{Y}^{(j)}$ 's. Then, if $k=2 n$ is even we let $\widehat{\mathcal{X}}_{k}$ be the vector field characterized by the property

$$
\left(\partial_{t_{k}}+z^{n}\right) \cdot W^{(\widehat{Y})} \subset W^{(\widehat{Y})},
$$

while if $k=2 n+1$ we let $\widehat{\mathcal{X}}_{k}$ be the vector field characterized by

$$
\left(\partial_{t_{k}}+\theta z^{n}\right) \cdot W^{(\widehat{Y})} \subset W^{(\widehat{Y})} .
$$

As for SCS, we can write down the equations defining $\widehat{\mathcal{X}}_{k}$ by comparing coefficients: if $k=2 n$

$$
\partial_{t_{k}} \widehat{Y}^{(j)}+z^{n} \widehat{Y}^{(j)}=\widehat{Y}^{(j+2 n)}+\sum_{l=1}^{n}\left(\widehat{Y}_{0, l}^{j} \widehat{Y}^{(2 n-2 l)}+\widehat{Y}_{1, l}^{j} \widehat{Y}^{(2 n-2 l+1)}\right),
$$

while if $k=2 n+1$

$$
\left\{\begin{array}{l}
\partial_{t_{k}} \widehat{Y}^{(2 j)}+\theta z^{n} \widehat{Y}^{(2 j)}=\widehat{Y}^{(2 j+2 n+1)}+\sum_{l=1}^{n} \widehat{Y}_{0, l}^{2 j} \widehat{Y}^{(2 n-2 l+1)} \\
\partial_{t_{k}} \widehat{Y}^{(2 j+1)}+\theta z^{n} \widehat{Y}^{(2 j+1)}=-\sum_{l=1}^{n} \widehat{Y}_{0, l}^{2 j+1} \widehat{Y}^{(2 n-2 l+1)}
\end{array}\right.
$$

The definition of $\widehat{\mathcal{Y}}_{k}$ is obtained imposing the further condition

$$
\begin{gathered}
\left(\partial_{t_{k}}+z^{n}\right) \cdot \hat{w} \in W^{(\widehat{Y})} \text { if } k=2 n, \\
\left(\partial_{t_{k}}+\theta z^{n}\right) \cdot \hat{w} \in W^{(\widehat{Y})} \text { if } k=2 n+1 .
\end{gathered}
$$

As in [10], we give the following 
Definition 4.1 We call super Sato System (SS) the family of vector fields $\left\{\widehat{\mathcal{X}}_{k}\right\}_{k>0}$ on $\widehat{\mathcal{M}}$ and super Darboux-Sato System $(S D S)$ the family of vector fields $\left\{\widehat{\mathcal{Y}}_{k}\right\}_{k>0}$ on $\widehat{\mathcal{N}}$.

The next step is to define the maps $\hat{\mu}: \widehat{\mathcal{N}} \rightarrow \widehat{\mathcal{M}}$ and $\hat{\sigma}: \widehat{\mathcal{N}} \rightarrow \widehat{\mathcal{P}}$. The first is as usual the projection

$$
\left(\left\{\widehat{Y}^{(k)}\right\}_{k \geq 0}, \hat{w}\right) \mapsto\left\{\widehat{Y}^{(k)}\right\}_{k \geq 0},
$$

while the second is defined by imposing the intertwining condition

$$
\hat{w} \cdot W=W^{(\widehat{Y})},
$$

which holds if and only if

$$
\left\{\begin{array}{l}
\hat{w} \widehat{H}^{(2 j)}=\widehat{Y}^{(2 j)}+\sum_{l=1}^{j}\left(\hat{w}_{0, l} \widehat{Y}^{(2 j-2 l)}+\hat{w}_{1, l} \widehat{Y}^{(2 j-2 l+1)}\right) \\
\hat{w} \widehat{H}^{(2 j+1)}=\widehat{Y}^{(2 j+1)}+\sum_{l=1}^{j} \hat{w}_{0, l} \widehat{Y}^{(2 j-2 l+1)}
\end{array}\right.
$$

Lemma 4.1 The SDS system is a Darboux intertwiner of SS with SCS.

Proof. We need only to prove that $\hat{\sigma}_{*}(S D S)=S C S$. This follows by observing that the definitions of SDS and $\hat{\sigma}$ imply

$$
\left\{\begin{array}{l}
\partial_{t_{2 k}} \hat{w}+z^{k} \hat{w}=\hat{w} \widehat{H}^{(2 k)} \\
\partial_{t_{2 k+1}} \hat{w}+\theta z^{k} \hat{w}=\widehat{H}^{(2 k+1)}
\end{array}\right.
$$

so

$$
\left\{\begin{array}{l}
\hat{w} \cdot\left(\partial_{t_{2 k}}+\widehat{H}^{(2 k)}\right)=\left(\partial_{t_{2 k}}+z^{k}\right) \cdot \hat{w} \\
\hat{w} \cdot\left(\partial_{t_{2 k+1}}+\widehat{H}^{(2 k+1)}\right)=\left(\partial_{t_{2 k}}+\theta z^{k}\right) \cdot \hat{w}
\end{array}\right.
$$

Hence, we get

$$
\begin{aligned}
\hat{w} \cdot\left(\partial_{t_{2 k}}+\widehat{H}^{(2 k)}\right) \cdot W & =\left(\partial_{t_{2 k}}+z^{k}\right) \cdot \hat{w} W \\
& =\left(\partial_{t_{2 k}}+z^{k}\right) \cdot W^{(\widehat{Y})} \subset W^{(\widehat{Y})}
\end{aligned}
$$

and

$$
\begin{aligned}
\hat{w} \cdot\left(\partial_{t_{2 k+1}}+\widehat{H}^{(2 k+1)}\right) \cdot W & =\left(\partial_{t_{2 k+1}}+\theta z^{k}\right) \cdot \hat{w} W \\
& =\left(\partial_{t_{2 k+1}}+\theta z^{k}\right) \cdot W^{(\widehat{Y})} \subset W^{(\widehat{Y})},
\end{aligned}
$$

showing that $\left(\partial_{t_{k}}+\widehat{H}^{(k)}\right) \cdot W \subset W$, i.e. the SCS.

We consider now the map $\hat{\rho}: \widehat{\mathcal{M}} \rightarrow \widehat{\mathcal{N}}$ defined by

$$
\left\{\widehat{Y}^{(k)}\right\}_{k \geq 0} \mapsto\left(\left\{\widehat{Y}^{(k)}\right\}_{k \geq 0}, \widehat{Y}^{(0)}\right)
$$

and the corresponding map $\hat{\sigma} \circ \hat{\rho}: \widehat{\mathcal{M}} \rightarrow \widehat{\mathcal{P}}$. 
Lemma 4.2 The submanifold $\hat{\rho}(\widehat{\mathcal{M}})$ of $\widehat{\mathcal{N}}$ is a section of $\hat{\mu}$ invariant under SDS.

Proof. The previous definitions imply

$$
\left\{\begin{array}{c}
\partial_{2 j}\left(\hat{w}-\widehat{Y}^{(0)}\right)=-z^{j}\left(\hat{w}-\widehat{Y}^{(0)}\right) \\
\quad+\sum_{l=1}^{j}\left(\left(\hat{w}_{0, l}-\widehat{Y}_{0, l}^{0}\right) \widehat{Y}^{(2 j-2 l)}+\left(\hat{w}_{1, l}-\widehat{Y}_{1, l}^{0}\right) \widehat{Y}^{(2 j-2 l+1)}\right) \\
\left.\partial_{2 j+1}\left(\hat{w}-\widehat{Y}^{(0)}\right)=-\theta z^{j}\left(\hat{w}-\widehat{Y}^{(0)}\right)+\sum_{l=1}^{j}\left(\hat{w}_{0, l}-\widehat{Y}_{0, l}^{0}\right)\right) \widehat{Y}^{(2 j-2 l+1)}
\end{array}\right.
$$

proving the lemma.

We have now to linearize the super Sato system. To achieve the result it is better to introduce the infinite even matrix $Y$ defined by

$$
\mathrm{Y}_{j k}:= \begin{cases}\widehat{Y}_{0, \frac{k+2}{2}}^{j} & \text { for } k \text { even } \\ \widehat{Y}_{1, \frac{k+1}{2}}^{j} & \text { for } k \text { odd },\end{cases}
$$

where $j, k \geq 0$, and the associated matrix $\widetilde{Y}$ whose entries are

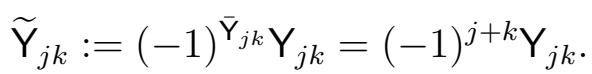

An easy computation shows that the flows of the SS hierarchy translate into the following Riccati type evolution equations:

$$
\left\{\begin{array}{l}
\partial_{2 n} \mathrm{Y}+\mathrm{Y} \Lambda_{2}^{t n}-\Lambda_{2}^{n} \mathrm{Y}=\mathrm{Y} \Gamma_{2 n} \mathrm{Y} \\
\partial_{2 n+1} \mathrm{Y}+\widetilde{\mathrm{Y}} \Lambda_{1} \Lambda_{2}^{t n}-\Lambda_{1} \Lambda_{2}^{n} \mathrm{Y}=\widetilde{\mathrm{Y}} \Gamma_{2 n+1} \mathrm{Y}
\end{array},\right.
$$

where ${ }^{t}$ means ordinary transposition (not super transposition), $\Lambda_{1}$ is the odd shift matrix with entries

$$
\left(\Lambda_{1}\right)_{j k}:=\frac{1-(-1)^{k}}{2} \delta_{k, j+1},
$$

$\Lambda_{2}$ is the even shift matrix with entries

$$
\left(\Lambda_{2}\right)_{j k}:=\delta_{k, j+2},
$$

$\Gamma_{2 n}$ is the even convolution matrix defined by

$$
\left(\Gamma_{2 n}\right)_{j k}:=\frac{1-(-1)^{k}}{2} \delta_{k, 2 n-j}+\frac{1-(-1)^{k+1}}{2} \delta_{k, 2 n-j-2}
$$

and finally $\Gamma_{2 n+1}$ is the odd convolution matrix given by

$$
\left(\Gamma_{2 n+1}\right)_{j k}:=\frac{1-(-1)^{k}}{2} \delta_{k, 2 n-j-1} .
$$

Observe that these matrices satisfy the relations

$$
\begin{gathered}
{\left[\Lambda_{1}, \Lambda_{1}\right]=\left[\Lambda_{1}, \Lambda_{2}\right]=\left[\Lambda_{1}, \Lambda_{2}^{t}\right]=0,} \\
\Lambda_{2}^{t} \Gamma_{n}=\Gamma_{n} \Lambda_{2}, \Lambda_{1} \Gamma_{2 n}=\Gamma_{2 n} \Lambda_{1}, \text { and } \Lambda_{1} \Gamma_{2 n+1}=\Gamma_{2 n+1} \Lambda_{1}=0,
\end{gathered}
$$

which imply the compatibility of the above system of matrix Riccati equations. 
Proposition 4.3 The infinite even matrix $Y$ is a solution of (4.1) if and only if it has the form $\mathrm{Y}=\mathrm{V} \cdot \mathrm{U}^{-1}$, where $\mathrm{U}$ and $\mathrm{V}$ are infinite even matrices satisfying the constant coefficients linear system

$$
\left\{\begin{array}{l}
\partial_{2 n} \mathrm{U}=\Lambda_{2}^{t n} \mathrm{U}-\Gamma_{2 n} \mathrm{~V} \\
\partial_{2 n+1} \mathrm{U}=\Lambda_{1} \Lambda_{2}^{t n} \mathrm{U}-\Gamma_{2 n+1} \mathrm{~V} \\
\partial_{2 n} \mathrm{~V}=\Lambda_{2}^{n} \mathrm{~V} \\
\partial_{2 n+1} \mathrm{~V}=\Lambda_{1} \Lambda_{2}^{n} \mathrm{~V}
\end{array}\right.
$$

with, of course, $\mathrm{U}$ invertible.

Proof. The proof is exactly the same as in the commutative case, once we have observed that for two matrices $\mathrm{U}$ and $\mathrm{V}$ the following relations hold:

$$
\left\{\begin{array}{l}
\partial_{t_{2 k}}(\mathrm{UV})=\left(\partial_{t_{2 k}} \mathrm{U}\right) \mathrm{V}+\mathrm{U}\left(\partial_{t_{2 k}} \mathrm{~V}\right) \\
\partial_{t_{2 k+1}}(\mathrm{UV})=\left(\partial_{t_{2 k+1}} \mathrm{U}\right) \mathrm{V}+\widetilde{\mathrm{U}}\left(\partial_{t_{2 k+1}} \mathrm{~V}\right) \\
\widetilde{\mathbf{U V}}=\widetilde{\mathbf{U}} \Rightarrow \widetilde{U}^{-1}=\widetilde{U}^{-1}
\end{array}\right.
$$

Thus, if $\mathrm{U}$ and $\mathrm{V}$ solve the system of linear equations of the statement and if we let $\mathrm{Y}=\mathrm{VU}^{-1}$, then

$$
\begin{aligned}
\partial_{2 n} \mathrm{Y} & =\left(\partial_{2 n} \mathrm{~V}\right) \mathrm{U}^{-1}-\mathrm{VU}^{-1}\left(\partial_{2 n} \mathrm{U}\right) \mathrm{U}^{-1} \\
& =\Lambda_{2}^{n} \mathrm{VU}^{-1}-\mathrm{VU}^{-1} \Lambda_{2}^{t n}+\mathrm{VU}^{-1} \Gamma_{2 n} \mathrm{VU}^{-1} \\
& =-\mathrm{Y} \Lambda_{2}^{t n}+\Lambda_{2}^{n} \mathrm{Y}+\mathrm{Y} \Gamma_{2 n} \mathrm{Y}
\end{aligned}
$$

and

$$
\begin{aligned}
\partial_{2 n+1} \mathrm{Y} & =\left(\partial_{2 n+1} \mathrm{~V}\right) \mathrm{U}^{-1}-\widetilde{\mathrm{V}} \widetilde{U}^{-1}\left(\partial_{2 n+1} \mathrm{U}\right) \mathrm{U}^{-1} \\
& =\Lambda_{1} \Lambda_{2}^{n} \mathrm{~V} \mathrm{U}^{-1}-\widetilde{\mathrm{V}} \widetilde{U}^{-1} \Lambda_{1} \Lambda_{2}^{t n}+\widetilde{\mathrm{V}} \widetilde{U}^{-1} \Gamma_{2 n+1} \mathrm{VU}^{-1} \\
& =-\widetilde{\mathrm{Y}} \Lambda_{1} \Lambda_{2}^{t n}+\Lambda_{1} \Lambda_{2}^{n} \mathrm{Y}+\widetilde{\mathrm{Y}} \Gamma_{2 n} \mathrm{Y}
\end{aligned}
$$

Therefore, if we look for a solution $Y$ of the Riccati matrix equations of SS with initial condition $Y(0)=Y_{0}$, we have simply to solve the linear system above imposing the initial conditions $\mathrm{V}(0)=\mathrm{Y}_{0}$ and $\mathrm{U}(0)=\mathbb{I}$. As we already noticed, the necessary condition

$$
\Gamma_{2 n+1} \Lambda_{1}=0
$$

for the integrability of the linear system holds.

Of course, the computations given in the proposition are only formal: to make sense of them one should also introduce a suitable notion of convergence for the intervening series in infinite variables. However, notice that the constraint " $Y_{j k}=0$ when either $j \geq J$ or $k \geq K$ " is compatible with the evolution equations for $\mathrm{Y}$, allowing us to consider reductions where only the finite submatrix $Y_{J K}$ of $Y$ consisting of its first 
$J$ rows and $K$ columns does not vanish. Obviously, $\mathrm{Y}_{J K}$ evolves according to the reduced Riccati equations

$$
\left\{\begin{array}{l}
\partial_{2 n} \mathrm{Y}_{J K}+\mathrm{Y}_{J K} \Lambda_{2, K K}^{t}-\Lambda_{2, J J}^{n} \mathrm{Y}_{J K}=\mathrm{Y}_{J K} \Gamma_{2 n, K J} \mathrm{Y}_{J K} \\
\partial_{2 n+1} \mathrm{Y}_{J K}+\widetilde{\mathrm{Y}}_{J K}\left(\Lambda_{1} \Lambda_{2}^{t}\right)_{K K}-\Lambda_{1, J J} \Lambda_{2, J J}^{n} \mathrm{Y}_{J K}=\widetilde{\mathrm{Y}}_{J K} \Gamma_{2 n+1, K J} \mathrm{Y}_{J K}
\end{array} .\right.
$$

This is a closed system of (graded) ordinary differential equations in a finite number of variables. It yields "finite type" solutions (i.e. depending only on finitely many times) of SS and hence of SCS and HSKP. Observe that the compatibility of the reduced system requires $K$ to be even; in this case $\left(\Lambda_{1} \Lambda_{2}^{t n}\right)_{K K}=\Lambda_{1, K K} \Lambda_{2, K K}^{t}{ }^{n}$.

\subsection{An explicit example}

To show an example, we compute the solution of SS associated to $J=3$ and $K=4$. To simplify notations let us call $Y:=\mathrm{Y}_{34}$,

$$
\begin{gathered}
A_{1}:=\Lambda_{1,33}=\left(\begin{array}{ccc}
0 & 1 & 0 \\
0 & 0 & 0 \\
0 & 0 & 0
\end{array}\right), \quad A_{2}:=\Lambda_{2,33}=\left(\begin{array}{lll}
0 & 0 & 1 \\
0 & 0 & 0 \\
0 & 0 & 0
\end{array}\right), \\
B_{1}:=\Lambda_{1,44}=\left(\begin{array}{cccc}
0 & 1 & 0 & 0 \\
0 & 0 & 0 & 0 \\
0 & 0 & 0 & 1 \\
0 & 0 & 0 & 0
\end{array}\right), \quad B_{2}:=\Lambda_{2,44}^{t}=\left(\begin{array}{llll}
0 & 0 & 0 & 0 \\
0 & 0 & 0 & 0 \\
1 & 0 & 0 & 0 \\
0 & 1 & 0 & 0
\end{array}\right),
\end{gathered}
$$

and $C_{k}:=\Gamma_{k, 43}$. The relevant (i.e. different from zero) convolution matrices are

$$
\begin{gathered}
C_{2}:=\left(\begin{array}{lll}
1 & 0 & 0 \\
0 & 1 & 0 \\
0 & 0 & 0 \\
0 & 0 & 0
\end{array}\right), \quad C_{3}:=\left(\begin{array}{lll}
0 & 1 & 0 \\
0 & 0 & 0 \\
0 & 0 & 0 \\
0 & 0 & 0
\end{array}\right), \\
C_{4}:=\left(\begin{array}{lll}
0 & 0 & 1 \\
0 & 0 & 0 \\
1 & 0 & 0 \\
0 & 1 & 0
\end{array}\right), \quad C_{5}:=\left(\begin{array}{lll}
0 & 0 & 0 \\
0 & 0 & 0 \\
0 & 1 & 0 \\
0 & 0 & 0
\end{array}\right), C_{6}:=\left(\begin{array}{lll}
0 & 0 & 0 \\
0 & 0 & 0 \\
0 & 0 & 1 \\
0 & 0 & 0
\end{array}\right) .
\end{gathered}
$$

We see that $A_{2}^{2}=0$ and $B_{2}^{2}=0$, so the solution of SS (or SCS) will depend only on the first six times. We solve the Riccati system for $Y$ by introducing the $4 \times 4$ matrix $U$ and the $3 \times 4$ matrix $V$ which are solutions of the following linear Cauchy problems:

$$
\left\{\begin{array} { l } 
{ \partial _ { t _ { 2 k } } V = A _ { 2 } ^ { k } V } \\
{ \partial _ { t _ { 2 k + 1 } } V = A _ { 1 } A _ { 2 } ^ { k } V } \\
{ V ( 0 ) = Y ( 0 ) }
\end{array} \quad \left\{\begin{array}{l}
\partial_{t_{2 k}} U=B_{2}^{k} U-C_{2 k} V \\
\partial_{t_{2 k+1}} U=B_{1} B_{2}^{k} U-C_{2 k+1} V \\
U(0)=\mathbb{I}
\end{array}\right.\right.
$$


and then putting $Y:=V U^{-1}$. First of all we find that

$$
V=\exp \sum_{j>0}\left(t_{2 j} A_{2}^{j}+t_{2 j-1} A_{1} A_{2}^{j-1}\right) V(0)=\left(\begin{array}{ccc}
1 & t_{1} & t_{2} \\
0 & 1 & 0 \\
0 & 0 & 1
\end{array}\right) Y(0) .
$$

Then we solve the system for $U$ by introducing the matrix $U_{0}$ defined by

$$
U=\exp \sum_{j>0}\left(t_{2 j} B_{2}^{j}+t_{2 j-1} B_{1} B_{2}^{j-1}\right) U_{0}=\left(\mathbb{I}+t_{1} B_{1}+t_{2} B_{2}+\left(t_{3}+t_{1} t_{2}\right) B_{1} B_{2}\right) U_{0}
$$

and evolving as

$$
\left\{\begin{array}{l}
\partial_{t_{2 k}} U_{0}=-\left(\mathbb{I}-t_{1} B_{1}-t_{2} B_{2}-\left(t_{3}-t_{1} t_{2}\right) B_{1} B_{2}\right) C_{2 k} V \\
\partial_{t_{2 k+1}} U_{0}=-\left(\mathbb{I}+t_{1} B_{1}-t_{2} B_{2}+\left(t_{3}-t_{1} t_{2}\right) B_{1} B_{2}\right) C_{2 k+1} V
\end{array} .\right.
$$

The equations for $U_{0}$ are easily solvable and we get

$$
U_{0}=\mathbb{I}-\left(\begin{array}{ccc}
t_{2} & t_{3} & t_{4}+\frac{1}{2} t_{2}^{2} \\
0 & t_{2} & 0 \\
t_{4}-\frac{1}{2} t_{2}^{2} & t_{5}-t_{2} t_{3} & t_{6}-\frac{1}{3} t_{2}^{3} \\
0 & t_{4}-\frac{1}{2} t_{2}^{2} & 0
\end{array}\right) Y(0)
$$

In order to write down an effective solution, we choose simple initial conditions, e.g.

$$
Y(0)=\left(\begin{array}{llll}
0 & 0 & 0 & 0 \\
0 & 0 & 0 & 0 \\
0 & 0 & 1 & 0
\end{array}\right)
$$

Then

$$
\begin{gathered}
V=\left(\begin{array}{cccc}
0 & 0 & t_{2} & 0 \\
0 & 0 & 0 & 0 \\
0 & 0 & 1 & 0
\end{array}\right), \\
U=\left(\begin{array}{cccc}
1 & t_{1} & -t_{4}-\frac{1}{2} t_{2}^{2} & 0 \\
0 & 1 & 0 & 0 \\
t_{2} & t_{3}+t_{1} t_{2} & 1-t_{6}-t_{2} t_{4}-\frac{1}{6} t_{2}^{3} & t_{1} \\
0 & t_{2} & 0 & 1
\end{array}\right) .
\end{gathered}
$$

Finally, we find

$$
\begin{gathered}
\widehat{Y}^{(0)}=1-\frac{3 t_{2}^{2}}{\tau} z^{-1}+\frac{3 t_{2}\left(t_{1} t_{2}-t_{3}\right)}{\tau} \theta z^{-1}+\frac{3 t_{2}}{\tau} z^{-2}-\frac{3 t_{1} t_{2}}{\tau} \theta z^{-2}, \\
\widehat{Y}^{(1)}=\theta \\
\widehat{Y}^{(2)}=z-\frac{3 t_{2}}{\tau} z^{-1}+\frac{3\left(t_{1} t_{2}-t_{3}\right)}{\tau} \theta z^{-1}+\frac{3}{\tau} z^{-2}-\frac{3 t_{1}}{\tau} \theta z^{-2}, \\
\widehat{Y}^{(2 k)}=z^{k} \quad \text { for } k>1
\end{gathered}
$$




$$
\widehat{Y}^{(2 k+1)}=\theta z^{k} \quad \text { for } k>0,
$$

where $\tau=3+t_{2}^{3}-3 t_{6}$. We can thus compute the first super currents of SCS

$$
\begin{gathered}
\widehat{H}^{(1)}=\theta+\sum_{k>0}\left(\frac{3 t_{2}^{2}}{\tau} z^{-1}+\frac{3 t_{2}}{\tau} z^{-2}\right)^{k} \theta \\
\widehat{H}^{(2)=}=z-\frac{3 t_{2}^{2}}{\tau}+3 \sum_{k \geq 0}\left(\frac{3 t_{2}^{2}}{\tau} z^{-1}-\frac{3 t_{2}}{\tau} z^{-2}\right)^{k} \times \\
\left(\frac{t_{2}^{2}}{\tau}-\frac{2 t_{2}}{\tau} z^{-1}+\frac{2 t_{1} t_{2}-t_{3}}{\tau} \theta z^{-1}+\frac{1}{\tau} z^{-2}-\frac{t_{1}}{\tau} \theta z^{-2}\right) .
\end{gathered}
$$

As explained in Section 2.2, we obtain a solution of HSKP after substituting $t_{2}$ and $t_{1}$ with $x$ and $\varphi+t_{1}$ respectively and putting $\hat{h}=\widehat{H}^{(1)}+\varphi \widehat{H}^{(2)}$ :

$$
\left\{\begin{array}{l}
\nu=0 \\
a=1+\sum_{k>0}\left(\frac{3 x^{2}}{\tau} z^{-1}-\frac{3 x}{\tau} z^{-2}\right)^{k} \\
h=z-\frac{6 x}{\tau} z^{-1}+\frac{3}{\tau} z^{-2}+3 \sum_{k>0}\left(\frac{3 x^{2}}{\tau} z^{-1}-\frac{3 x}{\tau} z^{-2}\right)^{k}\left(\frac{x^{2}}{\tau}-\frac{2 x}{\tau} z^{-1}+\frac{1}{\tau} z^{-2}\right) \\
\psi=3 \sum_{k \geq 0}\left(\frac{3 x^{2}}{\tau} z^{-1}-\frac{3 x}{\tau} z^{-2}\right)^{k}\left(\frac{2 t_{1} x-t_{3}}{\tau} z^{-1}-\frac{t_{1}}{\tau} z^{-2}\right) .
\end{array}\right.
$$

\section{Acknowledgments}

We would like to thank F. Magri for useful comments and suggestions. This work was partially supported by the GNFM branch of the Istituto Nazionale di Alta Matematica, and by the Italian MURST, under the Cofin99 project Geometry of Integrable Systems.

\section{References}

[1] H. Aratyn, Integrable Lax Hierarchies, their Symmetry Reductions and Multi-Matrix Models. Lectures at VIII J.A. Swieca Summer School, Section: Particles and Fields, Rio de Janeiro Brazil - February/95. LANL preprint, hep-th/9503211.

[2] M. J. Bergvelt and J. M. Rabin, Super Curves, their Jacobians, and Super KP Equations, alg-geom/9601012

[3] L. Bonora, Q. P. Liu, C. S. Xiong, The integrable hierarchy constructed from a pair of KdV-type hierarchies and its associated W-algebra. Comm. Math. Phys. 175 (1996), 177-202.

[4] P. Casati, G. Falqui, F. Magri and M. Pedroni, A Note on Fractional KdV Hierarchies, J. Math. Phys. 38 (1997) 4606-4628,

[5] P. Casati, G. Falqui, F. Magri and M. Pedroni, Darboux Coverings and Rational Reductions of the KP Hierarchy, Lett. Math. Phys. 41 (1997) 291-305 
[6] P. Casati, F. Magri, M. Pedroni, Bihamiltonian Manifolds and $\tau$-function. In: Mathematical Aspects of Classical Field Theory 1991 (M. J. Gotay et al. eds.), Contemporary Mathematics, Vol. 132, American Mathematical Society, Providence, R. I., (1992), pp. 213-234.

[7] E. Date, M. Jimbo, M. Kashiwara and T. Miwa, Transformation Groups for Soliton Equations, Proc. of RIMS Symposium on Nonlinear Integrable Systems - Classical Theory and Quantum Theory, M. Jimbo and T. Miwa eds., 39-119, World Scientific, Singapore, 1983

[8] L. A. Dickey, Soliton equations and Hamiltonian systems, Adv. Series in Math. Phys. 12, World Scientific, Singapore, 1991

[9] L. A. Dickey, On the constrained KP Hierarchy. I. LANL preprint, hep-th/9407038; II. LANL preprint, hep-th/9411005 and Lett. Math. Phys. 35 (1995), 229-236; II. An additional remark. LANL preprint, hep-th/9511157.

[10] G. Falqui, F. Magri and M. Pedroni, Bihamiltonian Geometry, Darboux Coverings, and Linearization of the KP Hierarchy, Commun. Math. Phys. 197 (1998) 303-324

[11] G. Falqui, F. Magri, M. Pedroni and J. P. Zubelli An Elementary Approach to the Polynomial $\tau$-functions of the KP Hierarchy. Proceedings of NEEDS98, Theor Math. Phys., to appear (2000).

[12] D. Friedan, Notes on String Theory and Two Dimensional Conformal Field Theory, in: M. Green and D. Gross eds., Unified String Theories, World Scientific, Singapore, 1986, pp. 162213

[13] I. M. Gel'fand and I. Zakharevich, On the local geometry of a bi-Hamiltonian structure, in: The Gelfand Mathematical Seminars 1990-1992 (L. Corwin et al. eds.), Birkäuser, Boston, 1993, pp. $51-112$

[14] I. M. Gel'fand and I. Zakharevich, The spectral theory for a pencil of skew-symmetrical operators of the third order. Comm. Pure Appl. Math. 47 (1994), 1031-1041.

[15] B. G. Konopelchenko, Nonlinear integrable equations: recursion operators, group theoretical and Hamiltonian structures of soliton equations, Springer Verlag, Berlin, 1987

[16] I. M. Krichever, General rational reductions of the KP hierarchy and their symmetries. Funct. Anal. Appl. 29 (1995), 75-80.

[17] B. A. Kupershmidt, Bosons and fermions interacting integrably with the Korteweg-de Vries field. J. Phys. A, 17 (1984), L869-L872.

[18] B. A. Kupershmidt, Canonical properties of the Miura maps between the mKP and KP hierarchies, continuous and discrete. Comm. Math. Phys. 167 (1995), 351-371

[19] A. Ibort, L. Martinez-Alonso, E. Medina-Reus, Explicit Solutions of supersymmetric KP hierarchies: supersolitons and solitinos. Jour. Math. Phys. 12 (1996), 6157-6172

[20] A. LeClair, Supersymmetric KP hierarchy: free field construction. Nucl. Phys. B 314 (1989), $425-438$

[21] Q.P. Liu, M. Manãs, Darboux transformation for the supersymmetric KdV equations. Lett. Math. Phys. 35 (1995), 115-122; Darboux transformation and supersymmetric KP hierarchy., solv-int/9909016. 
[22] F. Magri, M. Pedroni and J. P. Zubelli, On the Geometry of Darboux Transformations for the KP Hierarchy and its Connection With the Discrete KP Hierarchy, Commun. Math. Phys. 188 (1997) 305-325

[23] F. Magri and J. P. Zubelli, Bihamiltonian Formalism and the Darboux-Crum Method. Part I: From the KP to the mKP hierarchy, Inverse Probl. 13 (1997) 755-780

[24] Yu. I. Manin, Gauge Field Theory and Complex Geometry, Grund. Math. Wiss. 289, Springer Verlag, Heidelberg, 1988

[25] Yu. I. Manin and A. O. Radul, A Supersymmetric Extension of the Kadomtsev-Petviashvili Hierarchy, Commun. Math. Phys. 98 (1985) 65-77

[26] P. Mathieu, Supersymmetric extension of the Korteweg-de Vries equation. Jour. Math. Phys. 29 (1988), 2499-2506

[27] V. B. Matveev and M. A. Salle, Darboux transformations and solitons, Springer Verlag, New York, 1991

[28] M. Mulase, Algebraic Theory of the KP equations, in Perspectives in Mathematical Physics, 151-217, Conf. Proc. Lecture Notes Math. Phys. III, International Press, Cambridge, MA, 1994

[29] M. Mulase, Solvability of the super KP equation and a generalization of the Birkhoff decomposition, Invent. Math. 92 (1988) 1-46

[30] M. Mulase, A new Super KP System and a Characterization of the Jacobians of Arbitrary Algebraic Super Curves, J. Diff. Geom. 34 (1991) 651-680

[31] M. Mulase and J. M. Rabin, Super Krichever Functor, Int. J. Math. 2 (1991) 741-760

[32] J. M. Rabin, The Geometry of the Super KP Flows, Commun. Math. Phys. 137 (1991) 533-552

[33] M. Sato and Y. Sato, Soliton Equations as Dynamical Systems on Infinite-Dimensional Grassmann Manifold, in: P. Lax and H. Fujita (eds), Nonlinear PDEs in Applied Sciences (US-Japan Seminar, Tokyo), North Holland, Amsterdam, 1982, pp. 259-271

[34] A. S. Schwarz, Fermionic string and universal moduli space, Nucl. Phys. B317 (1989) 323-342

[35] G. Segal and G. Wilson, Loop Groups and Equations of KdV Type, Publ. Math. I.H.E.S. 61 (1985) 5-65

[36] M. Takama, Grassmannian Approach to Super-KP Hierarchies, hep-th/9506165

[37] K. Takasaki, Geometry of Universal Grassmann Manifold from Algebraic point of view, Rev. Math. Phys. 1 (1989) 1-46

[38] K. Ueno, H. Yamada, Super Kadomtsev-Petviashvili hierarchy and Super-Grassmann manifold. Lett. Math. Phys. 13 (1987), 59-68

[39] G. Wilson, On two Constructions of Conservation Laws for Lax equations. Quart. J. Math. Oxford 32 (1981), 491-512. 